Y. Hironaka and F. Sato

Nagoya Math. J.

Vol. 128 (1992), 121-151

\title{
FOURIER-EISENSTEIN TRANSFORM AND \\ PLANCHEREL FORMULA \\ FOR RATIONAL BINARY QUADRATIC FORMS
}

\section{YUMIKO HIRONAKA AND FUMIHIRO SATO}

\section{§ 0. Introduction}

0.1. Let $\boldsymbol{X}$ be the space of nondegenerate rational symmetric matrices of size 2 and put

$$
G=\left\{g \in G L_{2}(\mathbf{Q}) \mid \operatorname{det} g>0\right\} \text { and } \Gamma=S L_{2}(\mathbf{Z}) .
$$

The group $G$ acts on $\boldsymbol{X}$ by

$$
g * x=(\operatorname{det} g)^{-1} \cdot g x^{t} g .
$$

We are interested in the space $\mathscr{C}^{\infty}(\Gamma \backslash \boldsymbol{X})$ of $\Gamma$-invariant $\mathbf{C}$-valued functions on $\boldsymbol{X}$ and its subspace $\mathscr{\&}(\Gamma \backslash \boldsymbol{X})$ of functions whose supports consist of a finite number of $\Gamma$-orbits. The Hecke algebra $\mathscr{H}(G, \Gamma)$ of $G$ with respect to $\Gamma$ acts naturally on these spaces.

For an $x \in \boldsymbol{X}$, let $K=\mathbf{Q}(\sqrt{-\operatorname{det} x})$ or $\mathbf{Q} \oplus \mathbf{Q}$ according as $-\operatorname{det} x \notin$ $\left(\mathbf{Q}^{\times}\right)^{2}$ or $\in\left(\mathbf{Q}^{\times}\right)^{2}$. Take a positive rational number $r$ such that $r x$ is primitive half-integral and let $\mathfrak{f}(x)$ be the conductor of $r x$. For any positive integer $f$, denote by $\mathscr{O}_{f}^{1}$ the group of units with positive norm of the order of conductor $f$ of $K$. We define the Eisenstein series (zeta functions of binary quadratic forms) on $\boldsymbol{X}$ by

$$
E\left(x ; s_{1}, s_{2}\right)=\frac{1}{\mu(x)} \cdot \sum_{\substack{v \in \mathbf{Z}^{2} / S O(x) \\ v x^{t} v>0}} \frac{1}{\left|v x^{t} v\right|^{s_{1}+\frac{1}{2}}|\operatorname{det} x|^{s_{2}-\frac{1}{4}}},
$$

where $\mu(x)=\left[\mathscr{O}_{1}^{1}: \mathscr{O}_{f}^{1}(x)\right]$. As a function of $x$, the series $E\left(x ; s_{1}, s_{2}\right)$ is in $\mathscr{C}^{\infty}(\Gamma \backslash \boldsymbol{X})$ and will turn out to be a $\mathscr{H}(G, \Gamma)$-eigenfunction.

The purpose of the present paper is analysing the structure of $\mathscr{\&}(\Gamma \backslash \boldsymbol{X})$ as $\mathscr{H}(G, \Gamma)$-module through an integral transform with kernel function $E\left(x ; s_{1}, s_{2}\right)$, which we call the Fourier-Eisenstein transform.

Recieved April 8, 1992. 
0.2. Let $K=\mathbf{Q} \oplus \mathbf{Q}$ or a quadratic number field and $D=D_{K}$ the discriminant of $K$. We understand that $D=1$ if $K=\mathbf{Q} \oplus \mathbf{Q}$. For $r \in \mathbf{Q}, r>0$, we put

$$
X_{D, r}=\left\{x \in \boldsymbol{X} \mid \operatorname{det} x=-\frac{r^{2} D}{4}\right\}
$$

and, if $D<0$, we further put

$$
\begin{aligned}
& X_{D, r}^{+}=\left\{x \in \boldsymbol{X} \mid \operatorname{det} x=-\frac{r^{2} D}{4}, x \text { is positive definite }\right\} \\
& X_{\bar{D}, r}^{-}=\left\{x \in \boldsymbol{X} \mid \operatorname{det} x=-\frac{r^{2} D}{4}, x \text { is negative definite }\right\} .
\end{aligned}
$$

Then the $G$-orbit decomposition of $\boldsymbol{X}$ is given by

$$
\boldsymbol{X}=\left\{\bigsqcup_{D>0} \underset{\substack{r \in \mathbf{Q} \\ r>0}}{\bigsqcup} X_{D, r}\right\} \sqcup\left\{\bigsqcup_{D<0} \underset{\substack{r \in \mathbf{Q} \\ r>0}}{\bigsqcup}\left(X_{D, r}^{+} \sqcup X_{D, r}^{-}\right)\right\} .
$$

This yields the decomposition

$$
\mathscr{S}(\Gamma \backslash \boldsymbol{X})=\left\{\underset{D>0}{\bigoplus} \underset{\substack{r \in \mathbf{Q} \\
r>0}}{\bigoplus} \mathscr{S}\left(\Gamma \backslash X_{D, r}\right)\right\} \oplus\left\{\underset{\substack { D<0 \\
\begin{subarray}{c}{r \in \mathbf{Q} \\
r>0{ D < 0 \\
\begin{subarray} { c } { r \in \mathbf { Q } \\
r > 0 } }\end{subarray}}{\bigoplus}\left(\mathscr{S}\left(\Gamma \backslash X_{D, r}^{+}\right) \oplus \mathscr{S}\left(\Gamma \backslash X_{\bar{D}, r}^{-}\right)\right)\right\}
$$

into direct sum of $\mathscr{H}(G, \Gamma)$-submodules. Here we denote by $\mathscr{S}\left(\Gamma \backslash X_{D, r}^{( \pm)}\right)$the subspace of $\mathscr{S}(\Gamma \backslash \boldsymbol{X})$ consisting of functions whose supports are contained in $X_{D, r}^{( \pm)}$ For a fixed $D>0$ (resp. $D<0)$, all $\&\left(\Gamma \backslash X_{D, r}\right)$ (resp. $\left.\&\left(\Gamma \backslash X_{D, r}^{ \pm}\right)\right) \quad(r \in \mathbf{Q}$, $r>0)$ are isomorphic $\mathscr{H}(G, \Gamma)$-modules. Therefore it suffices to consider $\dot{\&}(\Gamma \backslash X)$, where $X=X_{D, 1}$ or $X_{D, 1}^{ \pm}$.

Let $\mathfrak{X}^{\mathrm{pr}}$ be the set of all primitive characters of the narrow ideal class groups of (not necessarily maximal) orders of $K$. Then we can define an orthogonal family of projections $\left\{p_{\chi} \mid \chi \in \mathfrak{X}^{\mathrm{pr}}\right\}$ of the $\mathscr{H}(G, \Gamma)$-module $\mathscr{S}(\Gamma \backslash X)$ and we have the direct sum decomposition

$$
\mathscr{S}(\Gamma \backslash X)=\bigoplus_{\chi \in \mathfrak{X}^{\mathrm{pr}}} \mathscr{S}(\Gamma \backslash X)_{\chi},
$$

where $\&(\Gamma \backslash X)_{\chi}=p_{\chi}(\&(\Gamma \backslash X))$.

Let

$$
\Re=\mathbf{C}\left[2^{t}+2^{-t}, 3^{t}+3^{-t}, \ldots, p^{t}+p^{-t}, \ldots\right],
$$

where $p$ runs over all rational primes. Define a homomorphism ${ }^{`}: \mathscr{H}(G, \Gamma) \rightarrow \Re$ by

$$
T(p, p)^{\wedge}=1, T(p, 1)^{-}=p^{1 / 2}\left(p^{t}+p^{-t}\right) \text { for any rational prime } p,
$$

where $T(p, p)$ and $T(p, 1)$ are the characteristic functions of $\Gamma\left(\begin{array}{ll}p & 0 \\ 0 & p\end{array}\right) \Gamma$ and 
$\Gamma\left(\begin{array}{ll}p & 0 \\ 0 & 1\end{array}\right) \Gamma$, respectively. We consider the ring $\Re$ as an $\mathscr{H}(G, \Gamma)$-module through this homomorphism. Then our main result (Theorem 3) is as follows:

Theorem. (i) For $\varphi \in \&(\Gamma \backslash X)_{\chi}$, put

$$
F_{\chi}(\varphi)(t)=a_{\chi}\left\{\sum_{x \in \Gamma \backslash X} \varphi(x) \mu(x) E(x ; t, 0)\right\} /\left(f_{\chi}\right)^{t} L\left(\chi ; t+\frac{1}{2}\right),
$$

where $L(\chi ; s)$ is the Hecke L-function attached to the class character $\chi$ and $a_{\chi}$ is a normalizing constant. Then $F_{\chi}(\varphi)(t)$ is contained in $\Re$ and the mapping

$$
F_{\chi}: \&(\Gamma \backslash X)_{\chi} \rightarrow \Re
$$

is an isomorphism of $\mathscr{H}(G, \Gamma)$-modules.

(ii) We have an $\mathscr{H}(G, \Gamma)$-module isomorphism

$$
\mathscr{\&}(\Gamma \backslash X)_{\chi} \cong \mathscr{H}(G, \Gamma) / \mathscr{I},
$$

where $\mathscr{I}$ is the ideal of $\mathscr{H}(G, \Gamma)$ generated by

$$
\{T(p, p)-1 \mid p: \text { rational primes }\}
$$

We define a structure of pre-Hilbert space on $\mathscr{S}(\Gamma \backslash X)_{\chi}$ via the inner product

$$
\langle\varphi, \phi\rangle_{s}=\sum_{x \in \Gamma \backslash X} \mu(x) \varphi(x) \overline{\phi(x)} .
$$

Let $L^{2}(\Gamma \backslash X)_{\chi}$ be the completion of $\mathscr{S}(\Gamma \backslash X)_{\chi}$. Moreover we construct a Hilbert space $\mathscr{L}_{\chi}^{2}$ which is a completion of $\Re$ with respect to an explicitly given inner product $\langle,\rangle_{\chi}$ and prove that the mapping $F_{\chi}$ can be extended to an isometry of $L^{2}(\Gamma \backslash X)$ onto $\mathscr{L}_{\chi}^{2}$ (Theorem 4 ). This result may be considered as the Plancherel formula for the (normalized) Fourier-Eisenstein transform $F_{\chi}$.

An explicit form of the inverse transformation of $F_{\chi}$ follows quite easily from the Plancherel formula (Theorem 5). Furthermore, using the main theorem, we can determine all $\mathscr{H}(G, \Gamma)$-eigenfunctions in $\mathscr{C}^{\infty}(\Gamma \backslash X)$ (Theorem 6).

0.3. Let $K$ be a real quadratic field. Then the set $K-\mathbf{Q}$ can naturally be identified with the space $X=X_{D, 1}$, where $D$ is the discriminant of $K$. The action of $G$ on $K-\mathbf{Q}$ is given by linear fractional transformation. Arakawa [A] and $\mathrm{Lu}$ [Lu] constructed certain $\mathscr{H}(G, \Gamma)$-eigenfunctions by arithmetic means. In $\S 5$, we shall dicuss these eigenfunctions from our point of view. 
0.4. In [M1] and [M2], Mautner took up the same problem for positive definite forms and obtained the decomposition (0.1). He further noted that $\mathscr{H}(G, \Gamma)$-eigenfunctions are products of local eigenfunctions. Our investigation can be viewed as a development of his work. We complete his results with the Plancherel formula, an explicit formula for eigenfunctions, the relation between eigenfunctions and zeta functions of binary quadratic forms, and a generalization to the case of indefinite forms.

0.5. In [SH], we have defined Eisenstein series and Fourier-Eisenstein transforms for reductive symmetric spaces and showed that analogous results can be obtained at least for the symmetric spaces $G L(n) \times G L(n) / \Delta(G L(n)), G L(2 n)$ $/ S p(n)$ and $G L(m+n) / G L(m) \times G L(n)$. Thus it is quite natural to expect that the results in the present paper will turn out to be one of the simplest examples of a general phenomenon.

Acknowledgement. We would like to express our gratitude to T. Arakawa for his helpful comments on the material in $\S 5$. A part of this work was done while the second author was staying in Göttingen under the support of the SFB 170.

\section{$\S 1$. Function spaces and the invariant measure on the set of rational binary quadratic forms}

1.1. Let

$$
\boldsymbol{X}=\left\{g \in G L_{2}(\mathbf{Q}) \mid{ }^{t} g=g\right\} \text { and } G=G L_{2}^{+}(\mathbf{Q})=\left\{g \in G L_{2}(\mathbf{Q}) \mid \operatorname{det} g>0\right\} .
$$

The group $G$ acts on $X$ by

$$
g * x=(\operatorname{det} g)^{-1} \cdot g x^{t} g .
$$

Put $\Gamma=S L_{2}(\mathbf{Z})$ and consider the following function spaces:

$$
\begin{aligned}
& \mathscr{C}^{\infty}(\Gamma \backslash \boldsymbol{X})=\{\varphi: \boldsymbol{X} \rightarrow \mathbf{C} \mid \varphi(\gamma * x)=\varphi(x), \text { for every } \gamma \in \Gamma\}, \\
& \mathscr{S}(\Gamma \backslash \boldsymbol{X})=\left\{\varphi \in \mathscr{C}^{\infty}(\Gamma \backslash \boldsymbol{X}) \mid \varphi=0 \text { outside a finite union of } \Gamma \text {-orbits }\right\}, \\
& \mathscr{C}^{\infty}(\Gamma \backslash Y)=\left\{\varphi \in \mathscr{C}^{\infty}(\Gamma \backslash \boldsymbol{X}) \mid \operatorname{Supp} \varphi \subset Y\right\}, \\
& \mathscr{S}(\Gamma \backslash Y)=\{\varphi \in \mathscr{S}(\Gamma \backslash \boldsymbol{X}) \mid \operatorname{Supp} \varphi \subset Y\}
\end{aligned}
$$

where $Y$ is a $G$-stable subset of $\boldsymbol{X}$. Denote by $\operatorname{ch}_{\Gamma g \Gamma}(g \in G)$ the characteristic function of the double coset $\Gamma g \Gamma$. As usual, the Hecke algebra $\mathscr{H}(G, \Gamma)$ of $G$ with respect to $\Gamma$ is defined to be the $\mathbf{C}$-vector space spanned by $\left\{\operatorname{ch}_{\Gamma g \Gamma} \mid g \in G\right\}$ with 
product

$$
\operatorname{ch}_{\Gamma g \Gamma} \cdot \operatorname{ch}_{\Gamma h \Gamma}=\sum_{\Gamma k \Gamma \in \Gamma \backslash G / \Gamma} m_{k} \operatorname{ch}_{\Gamma k \Gamma}
$$

where

$$
m_{k}=\#\left\{(i, j) \mid g_{i} h_{j} \in k \Gamma\right\}, \Gamma_{g} \Gamma=\bigsqcup_{i} g_{i} \Gamma, \Gamma h \Gamma=\bigsqcup_{j} h_{j} \Gamma .
$$

Here we use the symbol $\sqcup$ to indicate disjoint union. We define an action of $\mathscr{H}(G, \Gamma)$ on $\mathscr{C}^{\infty}(\Gamma \backslash \boldsymbol{X})$ by

$$
\left\{\operatorname{ch}_{\Gamma g \Gamma} * \varphi\right)(x)=\sum_{i} \varphi\left(g_{i}^{-1} * x\right), \text { where } \Gamma g \Gamma=\bigsqcup_{i} g_{i} \Gamma .
$$

Then, for any $G$-stable subset $Y$, the spaces $\mathscr{C}^{\infty}(\Gamma \backslash Y)$ and $\mathscr{\&}(\Gamma \backslash Y)$ are $\mathscr{H}(G, \Gamma)$-submodules of $\mathscr{C}^{\infty}(\Gamma \backslash \boldsymbol{X})$.

Our aim is to determine the $\mathscr{H}(G, \Gamma)$-module structure of $\mathscr{\&}(\Gamma \backslash \boldsymbol{X})$. For the discriminant $D$ of a quadratic field or $\mathbf{Q}$ and $r \in \mathbf{Q}, r>0$, put

$$
\begin{array}{ll}
X_{D, r}=\left\{x \in \boldsymbol{X} \mid \operatorname{det} x=-r^{2} D / 4\right\} & \text { if } D>0, \\
X_{D, r}^{+}=\left\{x \in \boldsymbol{X} \mid \operatorname{det} x=-r^{2} D / 4, x \text { is positive definite }\right\} & \text { if } D<0, \\
X_{D, r}^{-}=\left\{x \in \boldsymbol{X} \mid \operatorname{det} x=-r^{2} D / 4, x \text { is negative definite }\right\} & \text { if } D<0 .
\end{array}
$$

Then $G$ acts on these subsets transitively and we get the orbit decomposition

$$
\boldsymbol{X}=\left\{\bigsqcup_{D>0} \underset{\substack{r \in \mathbf{Q} \\ r>0}}{\bigsqcup} X_{D, r}\right\} \sqcup\left\{\bigsqcup_{D<0} \bigsqcup_{\substack{r \in \mathbf{Q} \\ r>0}}\left(X_{D, r}^{+} \sqcup X_{D, r}^{-}\right)\right\},
$$

and the direct sum decomposition

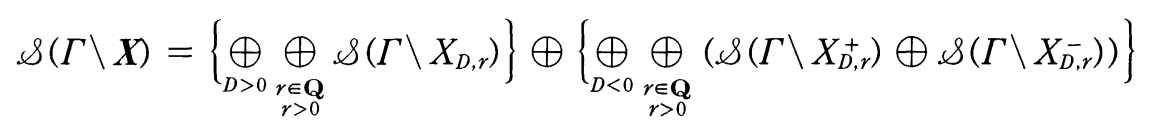

as $\mathscr{H}(G, \Gamma)$-module. Since $X_{D, r}=\left\{r x \mid x \in X_{D, 1}\right\}$ for $D>0$ and $X_{D, r}^{ \pm}=\{ \pm r x$ $\left.\mid x \in X_{D, 1}^{+}\right\}$for $D<0$, we have the following isomorphisms of $\mathscr{H}(G, \Gamma)$-modules:

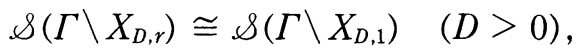

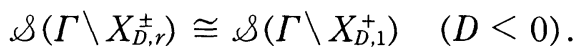

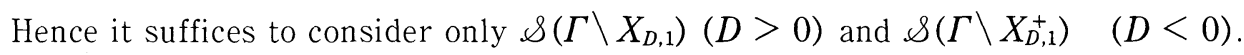

1.2. In the following, we always fix the discriminant $D$ of a quadratic field or $\mathbf{Q}$ and put $X=X_{D, 1}$ (resp. $\left.X_{D, 1}^{+}\right)$if $D>0$ (resp. $D<0$ ). We also put

$$
K=K_{D}= \begin{cases}\mathbf{Q} \oplus \mathbf{Q} & \text { if } D=1 \\ \mathbf{Q}(\sqrt{D}) & \text { if } D \neq 1\end{cases}
$$

We define the norm $N: K \rightarrow \mathbf{Q}$ by 


$$
N(x)= \begin{cases}x_{1} x_{2} & \text { if } D=1 \text { and } x=\left(x_{1}, x_{2}\right) \\ N_{K / \mathbf{Q}}(x) & \text { if } D \neq 1 .\end{cases}
$$

Let $\mathbf{P}$ be the set of rational primes. We define Dirichlet characters $\chi_{K}$ and $\chi_{K, f}$ with $f \in \mathbf{N}$ as follows: for $p \in \mathbf{P}$,

$$
\begin{aligned}
\chi_{K}(p) & =1 \quad \text { if } D=1, \\
\chi_{K}(p) & =\left\{\begin{aligned}
1 & \text { if } p \text { splits in } K \\
-1 & \text { if } p \text { is inert in } K \\
0 & \text { if } p \text { ramifies in } K
\end{aligned} \text { if } D \neq 1,\right. \\
\chi_{K, f}(p) & =\left\{\begin{array}{cc}
\chi_{K}(p) & \text { if } p \nmid f \\
0 & \text { if } p \mid f .
\end{array}\right.
\end{aligned}
$$

For each natural number $f$, let $\mathscr{O}_{f}$ be the $\mathbf{Z}$-order in $K$ of conductor $f$, i.e.

$$
\mathscr{O}_{f}= \begin{cases}\left\{(x, y) \in \mathbf{Z}^{2} \mid x \equiv y(\bmod f)\right\} & \text { if } D=1 \\ {\left[1, \frac{f(D+\sqrt{D})}{2}\right]} & \text { if } D \neq 1,\end{cases}
$$

and let

$$
\mathscr{O}_{f}^{1}=\left\{x \in \mathscr{O}_{f} \mid N(x)=1\right\} .
$$

We have used the symbol $[\alpha, \beta]$ to denote the $\mathbf{Z}$-lattice in $K$ with $\mathbf{Z}$-basis $\{\alpha, \beta\}$. For simplicity, we write $\mathscr{O}=\mathscr{O}$, and $\mathscr{O}^{1}=\mathscr{O}_{1}^{1}$.

For an $\mathscr{O}_{f}$-ideal $a$, we define its norm by $N_{f}(\mathfrak{a})=\left[\mathscr{O}_{f}: \mathfrak{a}\right]$. Then , for $\alpha \in \mathscr{O}_{f}$, we have $N_{f}\left(\alpha \mathscr{O}_{f}\right)=|N(\alpha)|$.

A full $\mathbf{Z}$-lattice $\mathfrak{a}$ in $\mathscr{O}$ is called an $\mathscr{O}_{f}$-proper ideal if $\{x \in K \mid \mathfrak{a} x \subseteq \mathfrak{a}\}=\mathscr{O}_{f}$. Let $I_{f}$ be the multiplicative semigroup of all $\mathscr{O}_{f}$-proper ideals. As usual, we write $\mathfrak{a} \sim \mathfrak{b}$ if $\mathfrak{b}=\mathfrak{a} x$ for some $x \in K$ with $N(x)>0$. Then the narrow ideal class group $C l_{f}$ is defined by $C l_{f}=I_{f} / \sim$. We denote by $h_{f}$ the order of $C l_{f}$. It is known that the class number $h_{f}$ is given explicitly by

$$
h_{f}=\frac{f h_{K}}{\left[\mathscr{O}^{1}: \mathscr{O}_{f}^{1}\right]} \Pi_{p \mid f}\left(1-\chi_{K}(p) p^{-1}\right) \text {, }
$$

where $h_{K}=h_{1}$ (cf. [L, Chapter 8, Theorem 7], for example). For $D=1$, it is easy to see that

$$
I_{f}=\{[(n, m t),(0, f t)] \mid n, t>0,0 \leq m<f,(f, m)=1, n \equiv m t(\bmod f)\}
$$

and

$$
C l_{f} \cong(\mathbf{Z} / f \mathbf{Z})^{\times}
$$


Now we recall the correspondence between the set of ideal classes and the set of equivalence classes of primitive binary quadratic forms. For $S, T \in \boldsymbol{X}$, we say that $S$ and $T$ are equivalent and write $S \sim T$ if $T=\gamma * S$ for some $\gamma \in \Gamma$. Put

$$
X_{f}^{\mathrm{pr}}=\left\{\left(\begin{array}{cc}
a & b / 2 \\
b / 2 & c
\end{array}\right) \mid a, b, c \in \mathbf{Z},(a, b, c)=1, b^{2}-4 a c=f^{2} D\right\} .
$$

Then

$$
X=\bigsqcup_{f \in \mathbf{N}} \frac{1}{f} X_{f}^{\mathrm{pr}}, \quad \frac{1}{f} X_{f}^{\mathrm{pr}}=\left\{\frac{1}{f} x \mid x \in X_{f}^{\mathrm{pr}}\right\} .
$$

We say that $x$ is of conductor $f$ if $x \in \frac{1}{f} X_{f}^{\mathrm{pr}}$. If $D=1$, a complete set of representatives of $X_{f}^{\mathrm{pr}} / \sim$ can be chosen as

$$
\left\{\left(\begin{array}{cc}
m & f / 2 \\
f / 2 & 0
\end{array}\right) \mid 0 \leq m<f,(f, m)=1\right\} .
$$

Then, as is well known, there is a bijective correspondence between $X_{f}^{\mathrm{pr}} / \sim$ and $C l_{f}$ induced by

$$
\begin{array}{ll}
\left(\begin{array}{cc}
m & f / 2 \\
f / 2 & 0
\end{array}\right) \mapsto[(m, m),(0, f)] & \text { if } D=1 . \\
\left(\begin{array}{cc}
a & b / 2 \\
b / 2 & c
\end{array}\right) \mapsto\left[a, \frac{b+f \sqrt{D}}{2}\right] & \text { if } D \neq 1,
\end{array}
$$

By this bijection we identify the both sets and use the following notation to indicate the corresponding classes:

$$
\begin{array}{ccc}
X_{f}^{\mathrm{pr}} / \sim & \leftrightarrow & C l_{f} \\
w & & w \\
{[S]} & \mapsto & {\left[\mathfrak{a}_{S}\right]} \\
{\left[S_{\mathfrak{a}}\right]} & \leftrightarrow & {[\mathfrak{a}] .}
\end{array}
$$

If $T \in X_{f}^{\mathrm{pr}}, S \in X_{f_{1}}^{\mathrm{pr}}$ and $f \mid f_{1}$, then $\mathfrak{a}_{T} \mathfrak{a}_{S}$ is an

$\mathscr{O}_{f}$-proper ideal. We denote by $T * S \in X_{f}^{\text {pr }}$ the matrix corresponding to $\mathfrak{a}_{T} \mathfrak{a}_{S}$, which is determined up to $\Gamma$-equivalence.

1.3. We recall the definition of the completions of $G$ and $X$ (cf. [SH]). Let $\Gamma(n)$ be the principal congruence subgroup of level $n \in \mathbf{N}$ : 


$$
\Gamma(n)=\{\gamma \in \Gamma \mid \gamma \equiv 1(\bmod n)\} .
$$

We define the completions $\tilde{G}$ and $\tilde{X}$ of $G$ and $X$, respectively, by

$$
\tilde{G}=\underset{\leftarrow}{\lim } G / \Gamma(n) \text { and } \tilde{X}=\underset{\leftarrow}{\lim } \Gamma(n) / X
$$

Then $\tilde{G}$ is a locally compact totally disconnected unimodular topological group and $\tilde{X}$ is a locally compact totally disconnected topological space. Since the action of $G$ on $X$ is uniquely extended to a continuous action of $\tilde{G}$ on $\tilde{X}$, we use the same symbol $*$ to denote the extended action.

We may identify $\tilde{G}$ with the closure of $G$ in $G L_{2}\left(\mathbf{A}_{f}\right)$, where $\mathbf{A}_{f}=\Pi_{p}^{\prime} \mathbf{Q}_{p}$, the finite part of the adele ring of $\mathbf{Q}$. In the present case, since the group $S L_{2}$ satisfies the strong approximation theorem, we have

$$
\tilde{G}=\left\{g \in G L_{2}\left(\mathbf{A}_{f}\right) \mid \operatorname{det} g \in \mathbf{Q} \text {, det } g>0\right\} .
$$

We denote by $\bar{\Gamma}$ the closure of $\Gamma$ in $\tilde{G}$. Then we get natural bijective correspondences between $\Gamma \backslash X$ and $\bar{\Gamma} \backslash \tilde{X}$, and between $\Gamma \backslash G / \Gamma$ and $\bar{\Gamma} \backslash \tilde{G} / \bar{\Gamma}$, so we may identify $\mathscr{C}^{\infty}(\Gamma \backslash X)$ with

$$
\mathscr{C}^{\infty}(\bar{\Gamma} \backslash \tilde{X})=\{\varphi: \tilde{X} \rightarrow \mathbf{C} \mid \varphi(\gamma * x)=\varphi(x), \gamma \in \bar{\Gamma}\},
$$

$\mathscr{\&}(\Gamma \backslash X)$ with

$$
\mathscr{S}(\bar{\Gamma} \backslash \tilde{X})=\left\{\varphi \in \mathscr{C}^{\infty}(\bar{\Gamma} \backslash \tilde{X}) \mid \varphi: \text { compactly supported }\right\}
$$

and $\mathscr{H}(G, \Gamma)$ with

$$
\mathscr{H}(\tilde{G}, \bar{\Gamma})=\left\{\begin{array}{l|l}
f: \tilde{G} \rightarrow \mathbf{C} & \begin{array}{l}
f: \text { compactly supported, } \\
f\left(\gamma_{1} x \gamma_{2}\right)=f(x)\left(\gamma_{1}, \gamma_{2} \in \Gamma\right)
\end{array}
\end{array}\right\} .
$$

We normalize the Haar measure $d g$ on $\tilde{G}$ by $\int_{\bar{\Gamma}} d g=1$. Then the multiplication of $\mathscr{H}(G, \Gamma)$ can be expressed as

$$
\left(f_{1} \cdot f_{2}\right)(h)=\int_{\tilde{G}} f_{1}(g) f_{2}\left(g^{-1} h\right) d g, \quad f_{1}, f_{2} \in \mathscr{H}(G, \Gamma)
$$

and the action of $\mathscr{H}(G, \Gamma)$ on $\mathscr{C}^{\infty}(\Gamma \backslash X)$ can be expressed as

$$
(f * \varphi)(x)=\int_{\tilde{G}} f(g) \varphi\left(g^{-1} * x\right) d g, \quad f \in \mathscr{H}(G, \Gamma), \varphi \in \mathscr{C}^{\infty}(\Gamma \backslash X) .
$$

By Proposition 2.6 of [SH], the space $\tilde{X}$ carries a $\tilde{G}$-invariant measure $d \mu$. For $x \in X$, denote by $\Gamma_{x}$ the isotropy subgroup of $\Gamma$ at $x$. We fix a base point $x_{0} \in X_{1}^{\mathrm{pr}}(\subset X)$ and we normalize $d \mu$ by setting 


$$
\int_{\overline{\Gamma * x_{0}}} d \mu=1
$$

Then, by Proposition 1.9 of [SH], we have

$$
\int_{\overline{\Gamma * x}} d \mu=\left[\Gamma_{x_{0}}: g_{x} \Gamma_{x} g_{x}^{-1}\right],
$$

where $g_{x} \in G$ for which $x_{0}=g_{x} * x$. For simplicity, we write $\mu(x)=\int_{\overline{\Gamma * x}} d \mu$. For later use, we compute the value of $\mu(x)$.

Lemma 1.1. If $x \in X$ is of conductor $f$, then $\mu(x)=\left[\mathscr{O}^{1}: \mathscr{O}_{f}^{1}\right]$. In particular, if $D=1$, then $\mu(x)=1$ for every $x \in X$.

Proof. Let $D=1$ and $x \in X$. Denote by $G_{x}^{(1)}$ the isotropy subgroup of $G^{(1)}=$ $S L_{2}(\mathbf{Q})$ at $x$. We may take $x_{0}=\left(\begin{array}{cc}0 & 1 / 2 \\ 1 / 2 & 0\end{array}\right)$ as base point. Then we get

$$
\Gamma_{x}=\Gamma \cap g_{x}^{-1} G_{x 0}^{(1)} g_{x}=\left\{ \pm\left(\begin{array}{ll}
1 & 0 \\
0 & 1
\end{array}\right)\right\} .
$$

Thus we get $\mu(x)=1$ by (1.2).

$$
\text { Let } \begin{aligned}
D \neq 1, x & =\frac{1}{f} S, S=\left(\begin{array}{cc}
a & b / 2 \\
b / 2 & c
\end{array}\right) \in X_{f}^{\mathrm{pr}} \text { and } x_{0}=T \in X_{1}^{\mathrm{pr}} \text {. Then } \\
G_{x}^{(1)}=G_{S}^{(1)} & =\left\{\left(\begin{array}{cc}
s & t \\
-\frac{c}{a} t & s+\frac{b}{a} t
\end{array}\right) \in G^{(1)} \mid(s, t) \in \mathbf{Q}^{2}-\{(0,0)\}\right\} .
\end{aligned}
$$

So we obtain an isomorphism

$$
\begin{aligned}
& \mathscr{O}_{f}^{1} \quad \rightarrow \quad \Gamma_{x} \\
& \frac{t+u f \sqrt{D}}{2} \mapsto\left(\begin{array}{cc}
\frac{t-b u}{2} & a u \\
-c u & \frac{t+b u}{2}
\end{array}\right)=S\left(\begin{array}{cc}
0 & u \\
-u & 0
\end{array}\right)+\left(\begin{array}{cc}
t / 2 & 0 \\
0 & t / 2
\end{array}\right) .
\end{aligned}
$$

Take a $g \in G$ such that $g * x=x_{0}$, equivalently $(f \operatorname{det} g) \cdot T=g S^{t} g$. Then we see 


$$
g \Gamma_{x} g^{-1}=\left\{T\left(\begin{array}{cc}
0 & u f \\
-u f & 0
\end{array}\right)+\left(\begin{array}{cc}
t / 2 & 0 \\
0 & t / 2
\end{array}\right) \mid \frac{t+u f \sqrt{D}}{2} \in \mathscr{O}_{f}^{1}\right\}
$$

while

$$
\Gamma_{x_{0}}=\left\{T\left(\begin{array}{cc}
0 & u \\
-u & 0
\end{array}\right)+\left(\begin{array}{cc}
t / 2 & 0 \\
0 & t / 2
\end{array}\right) \mid \frac{t+u \sqrt{D}}{2} \in \mathscr{O}^{1}\right\} .
$$

Now, by (1.2), it is obvious that $\mu(x)=\left[\mathscr{O}^{1}: \mathscr{O}_{f}^{1}\right]$.

\section{$\S 2$. Decomposition of $\&(\Gamma \backslash X)$ by characters of class groups}

For a positive integer $f$, let $\mathfrak{X}(f)$ be the character group of $C l_{f}$. If $f_{1} \mid f$, then there exists a canonical surjective homomorphism $p_{f_{1}}^{f}: C l_{f} \rightarrow C l_{f_{1}}$ induced by $\mathfrak{a} \mapsto$ $a \mathscr{O}_{f_{1}}$; hence we have a natural injective map

$$
\begin{aligned}
\operatorname{lnd}_{f}^{f_{1}} \mathfrak{X}\left(f_{1}\right) & \rightarrow \mathfrak{X}(f) \\
\psi & \stackrel{\psi}{f} \\
\chi & \mapsto \chi \chi^{\circ} p_{f_{1}}^{f} .
\end{aligned}
$$

The conductor $f_{\chi}$ of $\chi \in \mathfrak{X}(f)$ is defined by

$$
f_{\chi}=\min \left\{f_{1} \in \mathbf{N} \mid f_{1} \text { devides } f, \chi \in \operatorname{Ind}_{f}^{f_{1}}\left(\mathfrak{X}\left(f_{1}\right)\right)\right\} .
$$

If $f=f_{\chi}$, then $\chi \in \mathfrak{X}(f)$ is called primitive. Let $\mathfrak{X}^{\text {pr }}$ be the set of all primitive characters of arbitrary conductor.

Denote by $\operatorname{ch}_{x}$ the characteristic function of $\Gamma * x$ for $x \in X$. Let $\chi \in \mathfrak{X}^{\text {pr }}$ and $T \in X_{f}^{\text {pr }}$. Taking $f_{1}$ satisfying $f_{\chi} \mid f_{1}$ and $f \mid f_{1}$, we put

$$
p_{\chi}\left(\operatorname{ch} \frac{1}{f} T\right)=\frac{1}{h_{f_{1}}} \sum_{[S] \in C l f_{1}} \chi\left(p_{f_{\chi}}^{f_{1}}([S]) \operatorname{ch} \frac{1}{f}(T * S) .\right.
$$

It is easy to see that the right hand side is independent of the choice of such an $f_{1}$, hence we get a linear operator $p_{x}$ on $\mathscr{C}^{\infty}(\Gamma \backslash X)$. The operator $p_{x}$ stabilizes

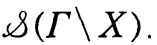

For a $\chi \in \mathfrak{X}^{\mathrm{pr}}$ and a positive integer $f$ such that $f_{\chi} \mid f$, set

$$
c_{\chi, f}=\frac{1}{h_{f}} \sum_{[S] \in C l_{f}} \chi\left(p_{f_{\chi}}^{f}([S]) \operatorname{ch} \frac{1}{f} s .\right.
$$

The purpose of this section is to show the following proposition. 
Proposition 2.1. (i) Let $\&(\Gamma \backslash X)_{\chi}=p_{\chi}(\&(\Gamma \backslash X))$. Then the space $\&(\Gamma \backslash$ $X)_{\chi}$ is spanned by $c_{\chi, f}\left(f \in \mathbf{N}, f_{\chi} \mid f\right)$.

(ii) The operators $p_{x}$ commute with the action of $\mathscr{H}(G, \Gamma)$ and we have an $\mathscr{H}(G, \Gamma)$-module isomorphism

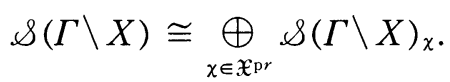

For the proof of Proposition 2.1, we need the following lemmas.

Lemma 2.2. For any $T \in X_{f}^{\mathrm{pr}}$, we have

$$
p_{\chi}\left(\operatorname{ch}_{\frac{1}{f} T}\right)= \begin{cases}0 & \text { if } f_{\chi} \nless f \\ \bar{\chi}([T]) c_{\chi, f} & \text { if } f_{\chi} \mid f,\end{cases}
$$

where

$$
\bar{\chi}([T])=\overline{\chi([T])} .
$$

Proof. It is easy to see that the identity holds for the case $f_{x} \mid f$. Let $f_{x} \backslash f$ and take a common multiple $f_{1}$ of $f$ and $f_{x}$. Then we have

$$
\begin{aligned}
& p_{\chi}\left(\operatorname{ch} \frac{1}{f} T\right)=\frac{1}{h_{f_{1}}} \sum_{[S] \in C l f} \operatorname{ch} \frac{1}{f}(T * S) \sum_{\substack{\left[l_{f} \in C l_{f_{1}} \\
p_{f}^{f 1}(U]\right)=[S]}} \chi\left(p_{f_{\chi}}^{f_{1}}([U])\right) \\
& =\frac{1}{h_{f_{1}}} \sum_{[S] \in C l f} \operatorname{ch} \frac{1}{f}(T * S) \chi\left(p_{f_{\chi}}^{f_{1}}\left(\left[U_{S}\right]\right)\right) \sum_{[V] \in \operatorname{Ker}\left(p_{f}^{f_{1}}\right)} \chi\left(p_{f_{\chi}}^{f_{1}}([V])\right),
\end{aligned}
$$

where $\left[U_{S}\right] \in C l_{f_{1}}$ with $p_{f}^{f_{1}}\left[U_{S}\right]=[S]$. Since $f_{\chi} \nmid f$, we get

$$
\sum_{[V] \in \operatorname{Ker}\left(p_{f}^{f_{1}}\right)} \chi\left(p_{f_{\chi}}^{f_{1}}([V])\right)=0
$$

hence

$$
p_{\chi}\left(\operatorname{ch} \frac{1}{f} T\right)=0 \text { if } f_{\chi} \times f .
$$

Lemma 2.3. (i) For any characters $\chi$ and $\phi$ in $\mathfrak{X}^{\mathrm{pr}}$, we have

$$
p_{\chi}^{\circ} p_{\phi}=p_{\psi^{\circ}} p_{\chi}=\delta_{\chi, \psi} p_{\chi},
$$

where $\delta_{x, \psi}$ is the Kronecker delta.

(ii) For any $S \in X_{f}^{\mathrm{pr}}$, we have

$$
\operatorname{ch}_{\frac{1}{f} S}=\sum_{\substack{x \in \mathfrak{x}_{\mathrm{pr}} \\ f_{\chi} \mid f}} \bar{\chi}\left(p_{f_{\chi}}^{f}([S])\right) c_{\chi, f} .
$$


Proof. Trivial from the orthogonality relation of characters.

Two lemmas above show that $\&(\Gamma \backslash X)_{\chi}=p_{\chi}(\&(\Gamma \backslash X))$ is spanned by

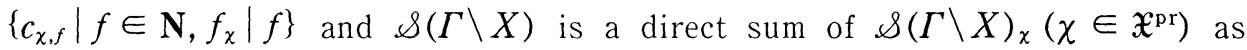
$\mathbf{C}$-vector space. Therefore, to prove Proposition 2.1, it suffices to show that the operators $p_{x}$ commute with the action of $\mathscr{H}(G, \Gamma)$. For this purpose it is convenient to introduce another $\mathscr{H}(G, \Gamma)$-action on $\mathscr{C}^{\infty}(\Gamma \backslash X)$. For $\varphi=\operatorname{ch}_{x} \in$ $\mathscr{C}^{\infty}(\Gamma \backslash X)$ and $f=\operatorname{ch}_{\Gamma g \Gamma} \in \mathscr{H}(G, \Gamma)$ with $\Gamma g \Gamma=\sqcup, \Gamma h_{\text {, }}$, put

$$
f * \varphi=\sum_{j} \operatorname{ch}_{h_{j} * x} .
$$

It is easy to see that (2.1) induces an $\mathscr{H}(\mathrm{G}, \Gamma)$-action on $\mathscr{C}^{\infty}(\Gamma \backslash X)$. Define a C-linear map $V: \mathscr{C}^{\infty}(\Gamma \backslash X) \rightarrow \mathscr{C}^{\infty}(\Gamma \backslash \mathrm{X})$ by $V\left(\operatorname{ch}_{x}\right)=\mu(x) \operatorname{ch}_{x}$.

Lemma 2.4. For every $f \in \mathscr{H}(G, \Gamma)$ and $\mathscr{C}^{\infty}(\Gamma \backslash X)$, the following identity holds:

$$
f * \varphi=V\left(f *\left(V^{-1} \varphi\right)\right) \text {. }
$$

Proof. We have only to show the identity for $f=\operatorname{ch}_{\Gamma g \Gamma} \in \mathscr{H}(G, \Gamma)$ and $\varphi=\operatorname{ch}_{x} \in \mathscr{C}^{\infty}(\Gamma \backslash X)$. Let

$$
\Gamma g \Gamma=\bigsqcup_{i} g_{i} \Gamma=\bigsqcup_{j} \Gamma h_{j}=\bigsqcup_{l} \Gamma m_{l} \Gamma_{x},
$$

where $\Gamma_{x}=\{\gamma \in \Gamma \mid \gamma * x=x\}$. Then we get

$$
\begin{aligned}
f * \varphi(y) & =\#\left\{i \mid y \in g_{i} \Gamma * x\right\} \\
& =\#\left\{i \mid \text { there exists } k \Gamma_{x} \in g_{\imath} \Gamma / \Gamma_{x} \text { such that } k * x=y\right\} \\
& =\#\left\{k \Gamma_{x} \in \Gamma g \Gamma / \Gamma_{x} \mid k * x=y\right\} .
\end{aligned}
$$

We see that the number of left $\Gamma_{x}$-cosets in $\Gamma m \Gamma_{x}$ which give the same element $r m * x$ in $X$ is equal to $\left[\Gamma_{m * x}: m \Gamma_{x} m^{-1} \cap \Gamma\right]$. So we obtain

$$
f * \varphi=\sum_{l}\left[\Gamma_{m_{l} * x}: m_{l} \Gamma_{x} m_{l}^{-1} \cap \Gamma\right] \mathrm{ch}_{m_{l} * x} .
$$

On the other hand we get

$$
f * \varphi=\sum_{l}\left[\Gamma_{x}: \Gamma_{x} \cap m_{l}^{-1} \Gamma m_{l}\right] \operatorname{ch}_{m_{l} * x},
$$

since the number of left $\Gamma$-cosets $\Gamma k$ satisfying $\Gamma h \Gamma_{x}=\Gamma k \Gamma_{x}$ is equal to $\left[\Gamma_{x}: \Gamma_{x} \cap h^{-1} \Gamma h\right]$. We obtain 


$$
\left[\Gamma_{x}: \Gamma_{x} \cap m^{-1} \Gamma m\right]=\left[m \Gamma_{x} m^{-1}: \Gamma_{m * x}\right]\left[\Gamma_{m * x}: m \Gamma_{x} m^{-1} \cap \Gamma\right]
$$

and, by (1.2),

$$
\left[m \Gamma_{x} m^{-1}: \Gamma_{m * x}\right]=\mu(m * x) / \mu(x) .
$$

Hence we get the required density.

By Lemma 1.1 and Lemma 2.2, we see that

$$
p_{x} \circ V=V \circ p_{x} .
$$

Hence the proof of the commutativity reduces to the proof of the identity

$$
p_{x}(f * \varphi)=f *\left(p_{x}(\varphi)\right), \quad f \in \mathscr{H}(G, \Gamma), \varphi \in \mathscr{C}^{\infty}(\Gamma \backslash X) .
$$

In the rest of this section, we consider the case $D \neq 1$, since the proof for $D=1$ is much easier. We need the following lemma due to Shintani (cf. [Sn, Lemmas 2.3 and 2.5]).

Lemma 2.5. Let $\mathfrak{a}$ be an $\mathscr{O}_{f}$-proper ideal and $p$ be a rational prime.

(i) Among $p+1$ sublattices in a of index $p$, there are $p-\chi_{K}(p) \mathscr{O}_{f p-p r o p e r}$ ideals and $1+\chi_{K}(p) \mathscr{O}_{f}$-proper ideals if $p \nmid f$, and there are $p \mathscr{O}_{f p}$-proper ideals and one $\mathscr{O}_{f / p}$-proper ideal if $p \mid f$.

Let $\left\{a_{1}, \ldots, a_{h_{f}}\right\}$ be a complete set of representatives of ideal classes in $C l_{f}$ and $\mathscr{B}$ the set of all sublattices of $a_{\imath}\left(1 \leq i \leq h_{f}\right)$ of index $p$.

(ii) For every $C \in C l_{f p}$, there are $\left[\mathscr{O}_{f}^{1}: \mathscr{O}_{f p}^{1}\right]$ lattices $\mathfrak{b}$ in $\mathscr{B}$ such that $\mathfrak{b}$ is $\mathscr{O}_{f p}$ proper and $\mathfrak{b} \in C$.

(iii) If $p \times f$, then for every $C \in C l_{f}$, there are $1+\chi_{K}(p)$ lattices $\mathfrak{b}$ in $\mathscr{B}$ such that $\mathfrak{b}$ is $\mathscr{O}_{f}$-proper and $\mathfrak{b} \in C$.

(iv) If $p \mid f$, then for every $C \in C l_{f / p}$, there are $h_{f} / h_{f / p}$ lattices $\mathfrak{b}$ in $\mathscr{B}$ such that is $\mathfrak{O}_{f / p}$-proper and $\mathfrak{b} \in C$.

Recall that $\mathscr{H}(G, \Gamma)$ is generated as a $\mathbf{C}$-algebra by the elements

$$
\left\{T(p, 1), T(p, p)^{ \pm 1} \mid p \in \mathbf{P}\right\},
$$

where $T(p, p)^{ \pm 1}$ (resp. $T(p, 1)$ ) is the characteristic function of the double $\Gamma$-coset containing $\left(\begin{array}{ll}p & 0 \\ 0 & p\end{array}\right)^{ \pm 1}\left(\operatorname{resp} .\left(\begin{array}{ll}p & 0 \\ 0 & 1\end{array}\right)\right)$. Since it is clear that the identity (2.2) holds for every $T(p, p)^{ \pm 1} \in \mathscr{H}(G, \Gamma)$, it suffices to show the following identity for every $p \in \mathbf{P}$ and $x \in \frac{1}{f} X_{f}^{\mathrm{pr}}$ : 


$$
p_{x}\left(T(p, 1) * \mathrm{ch}_{x}\right)=T(p, 1) * p_{x}\left(\mathrm{ch}_{x}\right) .
$$

We denote by $(R)$ (resp. $(L))$ the right (resp. left) hand side of (2.3).

Write $x=\frac{1}{f} S, S \in X_{f}^{\mathrm{pr}}$. First we consider the case where $f_{\chi} \Varangle f$. Then $(R)=0$ by Lemma 2.2. If $f_{\chi} \Varangle f p$, then clearly $(L)=0$. If $f_{\chi} \Varangle f p$, then we get by Lemma 2.5,

$$
\begin{aligned}
(L) & \left.=p_{\chi}\left(\left[\mathscr{O}_{f}^{1}: \mathscr{O}_{f p}^{1}\right] \sum_{[T]} \operatorname{ch}_{\frac{1}{f p} T}\right)\right) \\
& =\left[\mathscr{O}_{f}^{1}: \mathscr{O}_{f p}^{1}\right]\left(\sum_{[T]} \overline{\chi\left(p_{f_{\chi}}^{f_{p}}([T])\right)}\right) \cdot c_{\chi, f p},
\end{aligned}
$$

where the summation is taken over all $[T] \in C l_{f p}$ satisfying $p_{f}^{f p}([T])=[S]$. Since $f_{\chi} \nmid f$, we have $(L)=0$. Thus, we see that $(L)=(R)=0$ if $f_{\chi} \nmid f$.

Now we assume that $f_{\chi} \mid f$. The conductor of a lattice $\mathfrak{b}$ in $K$ is, by definition, a positive integer $f$ for which $\mathfrak{b}$ is an $\mathscr{O}_{f}$-proper ideal and we denote it by $\mathfrak{f}(\mathfrak{b})$. We may choose an ideal $a_{s}$ coprime to $p f$ from the ideal class corresponding to $S$. We get

$$
(L)=\sum_{\mathfrak{b}} \bar{\chi}(\mathfrak{b}) c_{\chi, \mathfrak{f}(\mathfrak{b})}
$$

where $\mathfrak{b}$ runs over all sublattices of $\mathfrak{a}_{S}$ of index $\boldsymbol{p}$ and

$$
\chi(\mathfrak{b})= \begin{cases}\chi\left(p_{f_{\chi}}^{\mathfrak{f}(\mathfrak{b})}([\mathfrak{b}])\right) & \text { if } f_{\chi} \mid \mathfrak{f}(\mathfrak{b}) \\ 0 & \text { if } f_{\chi} \times \mathfrak{f}(\mathfrak{b})\end{cases}
$$

We consider the right hand side $(R)$. For $[T] \in C l_{f^{\prime}}$ with $f_{\chi} \mid f^{\prime}$, we simply write $\chi(T)$ for $\chi\left(p_{f_{\chi}}^{f^{\prime}}[T]\right)$. Let

$$
(R)_{m}=\frac{\bar{\chi}(S)}{h_{f}} \sum_{[T] \in C l_{f}} \chi(T) \sum_{\mathfrak{b}} \operatorname{ch}_{\frac{1}{m} s_{\mathrm{b}}},
$$

where the summation with respect to $\mathfrak{b}$ is taken over all sublattices $\mathfrak{b}$ of $\mathfrak{a}_{T}$ satisfying $\left[a_{T}: \mathfrak{b}\right]=p$ and $\mathfrak{f}(\mathfrak{b})=m$. Then we see by Lemma 2.5 (i) that

$$
(R)= \begin{cases}(R)_{f p}+(R)_{f} & \text { if } p \nmid f \\ (R)_{f p}+(R)_{f / p} & \text { if } p \mid f .\end{cases}
$$

If $\mathfrak{b}$ is a sublattice of $\mathfrak{a}_{T}$ of index $p$ and $\mathfrak{f}(\mathfrak{b})=f p$, then $\mathfrak{b} \mathscr{O}_{f}=\mathfrak{a}_{T}$, and so we get by Lemma 2.5 (ii)

$$
\begin{aligned}
(R)_{f p} & =\bar{\chi}(S) \frac{h_{f p}\left[\mathscr{O}_{f}^{1}: \mathscr{O}_{f p}^{1}\right]}{h_{f}} c_{\chi, f p} \\
& =\left(p-\chi_{K, f}(p)\right) \bar{\chi}(S) \cdot c_{\chi, f p}
\end{aligned}
$$


(for the definition of $\chi_{K, f}$, see $\left.\$ 1.2\right)$. If $\mathfrak{b}$ is a sublattice of $a_{T}$ of index $p$ and $\mathfrak{f}(\mathfrak{b})=$ $f / p$, then $\mathfrak{b} \sim \mathfrak{a}_{T} \mathscr{O}_{f / p}$. Hence, if $p \mid f$, we get by Lemma 2.5 (iv)

$$
\begin{aligned}
(R)_{f / p} & =\frac{\bar{\chi}(S)}{h_{f}} \sum_{[U] \in C l_{f / p}} \operatorname{ch}_{f} \sum_{[T]} \chi(T) \\
& = \begin{cases}0 & \text { if } f_{\chi} \times \frac{f}{p} \\
\bar{\chi}(S) c_{\chi, f / p} & \text { if } f_{\chi} \mid \frac{f}{p},\end{cases}
\end{aligned}
$$

where the summation is taken over all $[T] \in C l_{f}$ such that $p_{f / p}^{f}([T])=[U]$. If $p \nmid f$, then we obtain, by Lemma 2.5 (iii),

$$
\begin{aligned}
& (R)_{f}=\frac{\bar{\chi}(S)}{h_{f}} \sum_{[T] \in C l f} \chi(T) \sum_{\substack{b \subset \vartheta_{f} \\
\left[\mathcal{O}_{f}:(b]=p, \bar{f}(b)=f\right.}} \operatorname{ch} \frac{1}{f}\left(T * S_{b}\right) \\
& =\sum_{\substack{b \subset a_{S} \\
\left[a_{s}: b\right]=p, f(b)=f}} \bar{\chi}(\mathfrak{b}) c_{\chi, f} .
\end{aligned}
$$

Hence we see that $(R)=(L)$, and this completes the proof of the commutativity, and so we finish the proof of Proposition 2.1.

\section{§3. Eisenstein series}

3.1. We define the Eisenstein series on $X$, which is a slight modification of the zeta functions of binary quadratic forms, by the following formula:

$$
E_{\varepsilon}\left(x ; s_{1}, s_{2}\right)=\mu(x)^{-1} \sum_{\substack{v \in \mathbf{Z}^{2} / \Gamma_{x} \\ v x^{\prime} v \neq 0}} \frac{\operatorname{sgn}^{\varepsilon}\left(v x^{t} v\right)}{\left|v x^{t} v\right|^{s_{1}+\frac{1}{2}}|\operatorname{det} x|^{s_{2}-\frac{1}{4}}},
$$

where $\varepsilon=0$ or $1, \operatorname{sgn}^{\varepsilon}()=\{\operatorname{sgn}()\}^{\varepsilon}$ and $\Gamma_{x}=\{\gamma \in \Gamma \mid \gamma * x=x\}$.

This Eisenstein series coincides with the one introduced in [SH, §3.1, (3.7)] up to the factor $\zeta\left(2 s_{1}+1\right)$ (see also [SH, §3.2]). The right hand side of (3.1) is absolutely convergent if $\operatorname{Re}\left(s_{1}\right)>\frac{1}{2}$, has a meromorphic continuation to the whole $\mathbf{C}^{2}$ and satisfies the following functional equation (cf. [S]):

$$
\Lambda_{\varepsilon}\left(x ; z_{2}, z_{1}\right)=\Lambda_{\varepsilon}\left(x ; z_{1}, z_{2}\right)
$$

where

$$
\Lambda_{\varepsilon}\left(x ; z_{1}, z_{2}\right)=\pi^{z_{1}-z 2} \Gamma\left(z_{2}-z_{1}+\frac{1}{2}\right) \eta_{D, \varepsilon}\left(z_{2}-z_{1}+\frac{1}{2}\right) E_{\varepsilon}\left(x ; z_{2}-z_{1},-z_{2}\right),
$$




$$
\eta_{D, \varepsilon}(s)=\left\{\begin{array}{cl}
1 & \text { if } D<0 \\
\cos (s \pi / 2) & \text { if } D>0 \text { and } \varepsilon=0 \\
\sin (s \pi / 2) & \text { if } D>0 \text { and } \varepsilon=1
\end{array}\right.
$$

3.2. As usual, for $S \in X_{f}^{\mathrm{pr}}$ and $s \in \mathbf{C}$, we define

$$
\zeta_{S}(s)=\sum_{\substack{v \in \mathbb{Z}^{2} / \Gamma_{s} \\ v S^{\prime} v>0}} \frac{1}{\left(v S^{t} v\right)^{s}}=\sum_{\substack{\alpha \in \mathfrak{a}_{s} / \theta_{s}^{1} \\ N(\alpha)>0}} \frac{N_{f}\left(\mathfrak{a}_{s}\right)^{s}}{N(\alpha)^{s}}
$$

and, for $C \in C l_{f}$ and $s \in \mathbf{C}$,

$$
\zeta^{(f)}(C ; s)=\sum_{\substack{a \in C \\ \mathfrak{a}+f \sigma_{f}=\theta_{s}}} \frac{1}{N(\mathfrak{a})^{s}} .
$$

The series $\zeta_{s}(s)$ and $\zeta^{(f)}(C ; s)$ are absolutely convergent for $\operatorname{Re}(s)>1$. It is obvious that $\zeta_{S}(s)$ depends only on $[S] \in C l_{f}$.

Theorem 1. Let $x=\frac{1}{f} S, S, \in X_{f}^{\mathrm{pr}}$. Then we have

$$
\begin{aligned}
E_{\varepsilon}(x ; s)= & f-\left(s_{1+\frac{1}{2}}\right)\left(\frac{D}{4}\right)^{-s_{2}+\frac{1}{4}} \\
& \times\left\{\begin{array}{cc}
\sum_{d \mid f} \frac{d^{2 s_{1}+1}}{\left[\mathscr{O}^{1}: \mathscr{O}_{d}^{1}\right]}\left(\zeta^{(d)}\left(p_{d}^{f}([S]) ; s_{1}+\frac{1}{2}\right)\right. & \\
\left.+(-1)^{\varepsilon} \zeta^{(d)}\left(p_{d}^{f}\left(J_{f} \cdot[S]\right) ; s_{1}+\frac{1}{2}\right)\right) & \text { if } D>0 \\
\sum_{d \mid f} \frac{d^{2 s_{1}+1}}{\left[\mathscr{O}^{1}: \mathscr{O}_{d}^{1}\right]} \zeta^{(d)}\left(p_{d}^{f}([S]) ; s_{1}+\frac{1}{2}\right) & \text { if } D<0,
\end{array}\right.
\end{aligned}
$$

where $J_{f}$ is the ideal class in $C l_{f}$ containing the ideal

$$
\begin{array}{ll}
(f \sqrt{D}) & \text { if } D>1 \\
((f,-f)) & \text { if } D=1 .
\end{array}
$$

Proof. From (3.1), (3.2) and Lemma 1.1, it is easy to see that

$$
E_{\varepsilon}(x ; s)=\frac{1}{\left[\mathscr{O}^{1}: \mathscr{O}_{f}^{1}\right]} f^{s_{1}+\frac{1}{2}}\left(\frac{D}{4}\right)^{-s_{2}+\frac{1}{4}} \times \begin{cases}\left(\zeta_{s}\left(s_{1}+\frac{1}{2}\right)+(-1)^{\varepsilon} \zeta_{s^{\prime}}\left(s_{1}+\frac{1}{2}\right)\right) \\ \zeta_{s}\left(s_{1}+\frac{1}{2}\right) & \text { if } D>0\end{cases}
$$


where $\left[S^{\prime}\right]=J_{f} \cdot[S]$. Hence the theorem is an immediate consequence of the following lemma.

Lemma 3.1. (i) Let $C \in C l_{f}$. If $\mathfrak{a} \in C$ satisfies $a+f \mathscr{O}_{f}=\mathscr{O}_{f}$, then

$$
\zeta^{(f)}(C ; s)=\sum_{\substack{\alpha \in \mathfrak{a} / O_{1}^{1} \\ N(\alpha)>0,(\alpha, f)=1}} \frac{N_{f}(\mathfrak{a})^{s}}{N(\alpha)^{s}}
$$

where $(\alpha, f)=1$ means $\alpha \mathscr{O}+f \mathscr{O}=\mathfrak{O}$.

(ii) For $S \in X_{f}^{\mathrm{pr}}$,

$$
\zeta_{S}(s)=\sum_{d \mid f}\left[\mathscr{O}_{d}^{1}: \mathscr{O}_{f}^{1}\right]\left(\frac{f}{d}\right)^{-2 s} \zeta^{(d)}\left(p_{d}^{f}([S]) ; s\right) .
$$

Proof. (i) We put

$$
\overline{\mathfrak{a}}=\{\bar{\alpha} \mid \alpha \in \mathfrak{a}\},
$$

where for $x \in K$,

$$
\bar{x}= \begin{cases}(b, a) & \text { if } D=1 \text { and } x=(a, b) \\ a-b \sqrt{D} & \text { if } D \neq 1 \text { and } x=a+b \sqrt{D}, a, b \in \mathbf{Q}\end{cases}
$$

Then we get

$$
N(\mathfrak{a})=N(\overline{\mathfrak{a}}) \text { and } \mathfrak{a} \overline{\mathfrak{a}}=N(\mathfrak{a}) \mathscr{O}_{f},
$$

and so

$$
\zeta^{(f)}(C ; s)=\zeta^{(f)}\left(C^{-1} ; s\right)
$$

There is a bijection

$$
\begin{array}{rlrl}
\{\alpha \in \mathfrak{a} \mid N(\alpha)>0,(\alpha, f)=1\} / \mathscr{O}_{f}^{1} & \rightarrow & \left\{\mathfrak{b} \in C^{-1} \mid \mathfrak{b}+f \mathscr{O}_{f}=\mathscr{O}_{f}\right\}, \\
& & \mapsto & \psi \\
\alpha & & \frac{\alpha \overline{\mathfrak{a}}}{N(\mathfrak{a})}
\end{array}
$$

and so we obtain the identity.

(ii) Let $a$ be an ideal belonging to the class $\left[\mathfrak{a}_{S}\right]$ such that $a+f \mathfrak{O}_{f}=\mathfrak{O}_{f}$. Then we see that

$$
\mathfrak{a} \mathscr{O}_{f_{1}} \cap \mathscr{O}_{f_{2}}=a \mathscr{O}_{f_{2}} \text { if } f_{1} \mid f_{2} \text { and } f_{2} \mid f
$$

and

$$
N_{f}(\mathfrak{a})=N_{f_{1}}\left(\mathfrak{a} \mathscr{O}_{f_{1}}\right) \text { if } f_{1} \mid f
$$


We see that, for $\alpha \in a-\{0\}$ and $d$ which divides $f$,

$$
(\alpha, f)_{\mathscr{O}} \subseteq d \mathscr{O} \text { if and only if } d^{-1} \alpha \in \mathfrak{a} \mathscr{O} \frac{f}{d} .
$$

For $d \mid f$, put

$$
\mathfrak{a}^{(d)}=\left\{\alpha \in \mathfrak{a} \mid(\alpha, f)_{\mathscr{O}}=d \mathscr{O}\right\},
$$

then

$$
a-\{0\}=\bigsqcup_{d \mid f} a^{(d)}
$$

Now we get

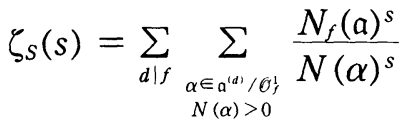

$$
\begin{aligned}
& =N_{f}(\mathfrak{a})^{s} \sum_{d \mid f} \sum_{\substack{\beta \in a \theta_{f, d} / \sigma_{l}^{1} \\
(\beta, f / d)=1, N(\beta)>0}} \frac{1}{N(d \beta)^{s}} \\
& =\sum_{d \mid f}\left[\mathscr{O}_{f / d}^{1}: \mathscr{O}_{f}^{1}\right] d^{-2 s} \zeta^{(f / d)}\left(\left[\mathfrak{a} \mathscr{O}_{f / d}\right] ; s\right) \\
& =\sum_{d \mid f}\left[\mathscr{O}_{d}^{1}: \mathscr{O}_{f}^{1}\right]\left(\frac{f}{d}\right)^{-2 s} \zeta^{(d)}\left(\left[a \mathscr{O}_{d}\right] ; s\right) .
\end{aligned}
$$

\section{§ 4. Fourier-Eisenstein transform and Plancherel formula}

4.1. Let $\mathscr{D} \&_{\varepsilon}$ be the $\mathbf{C}$-vector space of Dirichlet series

$$
\xi\left(z_{1}, z_{2}\right)=\sum_{m_{1}, m_{2} \in Q_{+}^{\star}} c\left(m_{1}, m_{2}\right) m_{1}^{-z_{1}} m_{2}^{-z_{2}}
$$

which converge absolutely for $\operatorname{Re}\left(z_{2}\right)-\operatorname{Re}\left(z_{1}\right)>\frac{3}{2}$, have meromorphic continuations to the whole $\mathbf{C}^{2}$ and satisfy the functional equation

$$
\Xi\left(z_{2}, z_{1}\right)=\Xi\left(z_{1}, z_{2}\right)
$$

where

$$
\Xi\left(z_{1}, z_{2}\right)=\pi^{z_{1}-z_{2}} \Gamma\left(z_{2}-z_{1}+\frac{1}{2}\right) \eta_{D, \varepsilon}\left(z_{2}-z_{1}+\frac{1}{2}\right) \xi\left(z_{1}, z_{2}\right) .
$$

We define the Fourier-Eisenstein transform on $\mathscr{S}(\Gamma \backslash X)$ as follows: 


$$
\begin{aligned}
& F_{\varepsilon}: \mathscr{Q}(\Gamma \backslash X) \rightarrow \mathscr{D} \mathscr{Q}_{\varepsilon} \\
& \psi \omega \\
& \varphi \quad \mapsto F_{\varepsilon}(\varphi)(s)=\int_{\tilde{X}} \varphi(x) E_{\varepsilon}\left(x ; s_{1}, s_{2}\right) d \mu(x) .
\end{aligned}
$$

Here we consider $E_{\varepsilon}(x ; s)$ as a function in $\mathscr{C}^{\infty}(\Gamma \backslash X)$. Note that $F_{\varepsilon}(\varphi)(s)$ is a finite linear combination of the Eisenstein series. In fact, by Lemma 1.1, we have

$$
F_{\varepsilon}(\varphi)(s)=\sum_{x \in \Gamma \backslash X} \varphi(x)\left[\mathscr{O}^{1}: \mathscr{O}_{\mathrm{f}(x)}^{1}\right] E_{\varepsilon}\left(x ; s_{1}, s_{2}\right),
$$

where $\mathfrak{f}(x)$ is the conductor of $x$. Hence $F_{\varepsilon}(\varphi)$ is in $\mathscr{D} \mathscr{S}_{\varepsilon}$.

Let

$$
\Re=\mathbf{C}\left[x_{2}, x_{3}, \ldots, x_{p}, \ldots\right], \quad x_{p}=p^{t}+p^{-t}(p \in \mathbf{P}) .
$$

Proposition 4.1. (i) There is a surjective $\mathbf{C}$-algebra homomorphism

$$
\begin{array}{rll}
\mathscr{H}(G, \Gamma) & \rightarrow & \Re \\
\psi & & \\
f & \mapsto & \hat{f}(t)=\int_{\tilde{G}}\left|\frac{a(p(g))}{d(p(g))}\right|_{\mathbf{A}_{f}}^{t+1 / 2} f(g) d g,
\end{array}
$$

where $p(g)$ is an element in $\left\{\left(\begin{array}{ll}a & b \\ c & d\end{array}\right) \in \tilde{G} \mid b=0\right\}$ such that $g p(g)^{-1} \in \bar{\Gamma}$ and $a(p(g))$ and $d(p(g))$ are the $(1,1)$-entry and the $(2,2)$-entry of $p(g)$, respectively.

(ii) The following identities hold for any $f \in \mathscr{H}(G, \Gamma)$ and any $\varphi \in \mathscr{S}(\Gamma \backslash X)$ :

$$
\begin{aligned}
F_{\varepsilon}(f * \varphi)(s) & =\hat{f}\left(s_{1}\right) F_{\varepsilon}(\varphi)(s) \\
\left(f * E_{\varepsilon}\right)\left(x ; s_{1}, s_{2}\right) & =\hat{f}\left(s_{1}\right) E_{\varepsilon}\left(x ; s_{1}, s_{2}\right) .
\end{aligned}
$$

Proof. (i) By the Iwasawa decomposition of $G L(2)$, we see that $f \mapsto \hat{f}$ is a C-algebra homomorphism. By direct computation, we get

$$
T(p, p)^{\wedge}(t)=1 \text { and } T(p, 1)^{\wedge}(t)=p^{1 / 2}\left(p^{t}+p^{-t}\right) .
$$

Thus we obtain the result.

(ii) The former identity is an immediate consequence of [SH, Theorem 2]. Since

$$
F_{\varepsilon}\left(\operatorname{ch}_{x}\right)(s)=\mu(x) E_{\varepsilon}(x, s),
$$

we obtain

$$
\left(f * E_{\varepsilon}\right)(x ; s)=\mu(x)^{-1} F_{\varepsilon}\left(f^{\iota} * \mathrm{ch}_{x}\right)(s),
$$

where $f^{\prime}(g)=f\left(g^{-1}\right)$. It is easy to see that $f^{\iota} * \varphi=f * \varphi$ for any $f \in \mathscr{H}(G$, 
$\Gamma)$ and $\varphi \in \mathscr{S}(\Gamma \backslash X)$. Hence

$$
\begin{aligned}
\left(f * E_{\varepsilon}\right)(x ; s) & =\mu(x)^{-1} \hat{f}\left(s_{1}\right) F_{\varepsilon}\left(\operatorname{ch}_{x}\right)(s) \\
& =\hat{f}\left(s_{1}\right) E_{\varepsilon}(x ; s) .
\end{aligned}
$$

This concludes the proof.

Remark. The homomorphism given in the first part of the proposition above is nothing but a specialization of (the tensor product of) the Satake transform on $G L(2)$. We call the homomorphism the restricted Satake transform.

Let $\chi \in \mathfrak{X}^{\mathrm{pr}}$ and $\varphi \in \mathscr{S}(\Gamma \backslash X)_{\chi}$, and define the normalized FourierEisenstein transform $F_{\chi}$ by

$$
F_{\chi}(\varphi)(t)=\frac{\sum_{\varepsilon=0,1} F_{\varepsilon}(\varphi)\left(t, s_{2}\right)}{\sum_{\varepsilon=0,1} F_{\varepsilon}\left(c_{\chi, f_{\chi}}\right)\left(t, s_{2}\right)} .
$$

It is obvious that the right hand side of the identity is independent of $s_{2}$.

Theorem 2. For an $m \in \mathbf{N}$, define a function $\phi_{x, m}(t) \in \Re$ by setting

$$
\begin{aligned}
& \phi_{x, m}(t)=\prod_{p \mid m} \phi_{\chi, p^{e_{p}}}(t), \quad e_{p}=\operatorname{ord}_{p}(m), \\
& \phi_{x, p e}(t)=\left\{\begin{array}{lc}
p^{-\frac{e}{2}} p^{(e+1) t}-p^{-(e+1) t} & \text { if } \chi_{K, f_{x}}(p)=0 \\
\frac{p^{t}-p^{-t}}{\left(1+p^{-1}\right)\left(p^{t}-p^{-t}\right)}\left\{p^{(e-1) t}\left(p^{2 t}-p^{-1}\right)-p^{-(e-1) t}\left(p^{-2 t}-p^{-1}\right)\right\} \\
\frac{p^{-\frac{e}{2}}}{\left(1-p^{-1}\right)\left(p^{t}-p^{-t}\right)}\left\{p ^ { e t } \left(p^{t}+p^{-1-t}-(\chi(p)+\bar{\chi}(p)) p^{\left.-\frac{1}{2}\right)}\right.\right. \\
-p^{-e t}\left(p^{-t}+p^{-1+t}-(\chi(p)+\bar{\chi}(p)) p^{\left.\left.-\frac{1}{2}\right)\right\}}\right. & \text { if } \chi_{K, f_{x}}(p)=1,
\end{array}\right.
\end{aligned}
$$

where

$$
\chi(\mathfrak{p})= \begin{cases}\chi\left(\left[(p, p),\left(1, f_{\chi}\right)\right]\right) & \text { if } D=1 \\ \chi\left(\left[\mathfrak{p} \cap \mathscr{O}_{f_{x}}\right]\right) & \text { if } D \neq 1 \text { and }(p)=\mathfrak{p} \bar{p} \text { in } K .\end{cases}
$$

Then, for $f_{\chi} \mid f$, we have

$$
F_{\chi}\left(c_{\chi, f}\right)(t)=\left[\mathscr{O}_{f_{\chi}}^{1}: \mathscr{O}_{f}^{1}\right] \phi_{\chi, f / f_{\chi}}(t) .
$$

In particular, for any $\varphi \in \&(\Gamma \backslash X)_{\chi}, F_{\chi}(\varphi)$ is contained in the ring $\Re$. 
For the proof of the theorem above, we prepare some notation on $L$-functions of quadratic fields. For $\chi \in \mathfrak{X}(f)$ and $s \in \mathbf{C}$, let

$$
L^{(f)}(\chi ; s)=\sum_{C \in C l_{f}} \chi(C) \zeta^{(f)}(C ; s)
$$

and

$$
L(\chi ; s)=L^{\left(f_{\chi}\right)}(\chi ; s) .
$$

Then, if $\operatorname{Re}(s)>1$, we obtain

$$
L(\chi ; s)=\prod_{p} L_{p}(\chi ; s) \text { and } L^{(f)}(\chi ; s)=L(\chi ; s) / \prod_{p \mid f} L_{p}(\chi ; s),
$$

where

$$
L_{p}(\chi ; s)=\left\{\begin{array}{cl}
\prod_{\substack{p: \text { prime in } K \\
p \in \mathfrak{p}}} \frac{1}{1-\chi\left(\left[\mathfrak{p} \cap \mathscr{O}_{f}\right]\right) N(\mathfrak{p})^{-s}} & \text { if } p \nmid f_{\chi} \text { and } D \neq 1 \\
\frac{1}{\left(1-\chi([p]) p^{-s}\right)\left(1-\overline{\chi([p])} p^{-s}\right)} & \text { if } p \nmid f_{\chi} \text { and } D=1 \\
1 & \text { if } p \mid f_{\chi} .
\end{array}\right.
$$

Here we write $\chi([p])$ for $\chi\left(\left[(p, p),\left(1, f_{\chi}\right)\right]\right)$.

Proof of Theorem 2. Let $f_{\chi} \mid f$ and put $\sigma=0$ or 1 according as $D<0$ or $D>0$. Then, by Theorem 1 , we obtain

$$
\begin{aligned}
& F_{\varepsilon}\left(c_{\chi, f}\right)(s)=\frac{\left[\mathscr{O}^{1}: \mathscr{O}_{f}^{1}\right]}{h_{f}} \sum_{[S] \in C l_{f}} \chi\left(p_{f_{x}}^{f}([S]) E_{\varepsilon}\left(\frac{1}{f} S ; s\right)\right. \\
& \left.=\frac{f^{-\left(s_{1}+\frac{1}{2}\right)}\left(\frac{D}{4}\right)^{-s 2+\frac{1}{4}}}{h_{f}} \sum_{d \mid f} \mid \mathfrak{O}_{d}^{1}: \mathscr{O}_{f}^{1}\right] d^{2 s_{1}+1} \\
& \times \sum_{[S] \in C l f} \chi\left(p _ { f _ { x } } ^ { f } ( [ S ] ) \left\{\zeta^{(d)}\left(p_{d}^{f}([S]) ; s_{1}+\frac{1}{2}\right)\right.\right. \\
& \left.+(-1)^{\varepsilon} \sigma \cdot \zeta^{(d)}\left(p_{d}^{f}\left(J_{f} \cdot[S]\right) ; s_{1}+\frac{1}{2}\right)\right\} \\
& \left.=\frac{f^{-\left(s_{1}+\frac{1}{2}\right)}\left(\frac{D}{4}\right)^{-s_{2}+\frac{1}{4}}}{h_{f}} \sum_{d \mid f} \mid \mathscr{O}_{d}^{1}: \mathscr{O}_{f}^{1}\right] d^{2 s_{1}+1} \sum_{[T] \in C l d} \zeta^{(d)}\left([T] ; s_{1}+\frac{1}{2}\right) \\
& \sum_{\substack{[S] \in C l f \\
p_{d}^{f}([S])=[T]}}\left\{\chi\left(p_{f_{x}}^{f}([S])\right)+(-1)^{\varepsilon} \sigma \cdot \chi\left(p_{f_{x}}^{f}\left(J_{f} \cdot[S]\right)\right)\right\}
\end{aligned}
$$




$$
\begin{aligned}
= & \left(1+(-1)^{\varepsilon} \sigma \cdot \bar{\chi}\left(J_{f_{\chi}}\right)\right) f^{-s_{1}-\frac{1}{2}}\left(\frac{D}{4}\right)^{-s_{2}+\frac{1}{4}} \\
& \times \sum_{\substack{d \mid f \\
f_{\chi} \backslash d}} \frac{\left[\mathscr{O}_{d}^{1}: \mathscr{O}_{f}^{1}\right] d^{2 s_{1}+1}}{h_{d}} L^{(d)}\left(\operatorname{Ind}_{d}^{f_{\chi}}(\chi) ; s_{1}+\frac{1}{2}\right) .
\end{aligned}
$$

By (1.1), we obtain

$$
\begin{aligned}
F_{\varepsilon}\left(c_{\chi, f}\right)(s) & =\left(1+(-1)^{\varepsilon} \sigma \cdot \bar{\chi}\left(J_{f_{x}}\right)\right) \frac{\left[\mathscr{O}^{1}: \mathscr{O}_{f}^{1}\right]}{h_{K}} f^{-s_{1}-\frac{1}{2}}\left(\frac{D}{4}\right)^{-s_{2}+\frac{1}{4}} \\
& \times L\left(\chi ; s_{1}+\frac{1}{2}\right) \sum_{\substack{d\left|f \\
f_{\chi}\right| d}} d^{2 s_{1}} \prod_{p \mid d} \frac{L_{p}^{-1}\left(\chi ; s_{1}+\frac{1}{2}\right)}{1-\chi_{K}(p) p^{-1}} .
\end{aligned}
$$

Hence we get

$$
\begin{aligned}
F_{\chi}\left(c_{\chi, f}\right)(t) & =\left[\mathscr{O}_{f_{\chi}}^{1}: \mathscr{O}_{f}^{1}\right]\left(\frac{f}{f_{\chi}}\right)^{-t-\frac{1}{2}} \sum_{d \mid \frac{f}{f_{\chi}}} d^{2 t} \prod_{p \mid d} \frac{L_{p}^{-1}\left(\chi ; t+\frac{1}{2}\right)}{1-\chi_{K, f_{\chi}}(p) p^{-1}} \\
& =\left[\mathscr{O}_{f_{\chi}}^{1}: \mathscr{O}_{f}^{1}\right] \prod_{p \mid \frac{f}{f_{\chi}}} p^{-e_{p}\left(t+\frac{1}{2}\right)}\left(1+\frac{L_{p}^{-1}\left(\chi ; t+\frac{1}{2}\right)}{1-\chi_{K, f_{\chi}}(p) p^{-1}} \sum_{n=1}^{e p} p^{2 t n}\right),
\end{aligned}
$$

where $e_{p}=\operatorname{ord}_{p}\left(f / f_{\chi}\right)$. By (4.6), we obtain the identity (4.4).

Through the restricted Satake transform ${ }^{`}: \mathscr{H}(G, \Gamma) \rightarrow \Re$ given in Proposition 4.1 (i), we consider the ring $\Re$ as an $\mathscr{H}(G, \Gamma)$-module. Then, by (4.2) and Theorem 2, the normalized Fourier-Eisenstein transform $F_{\chi}$ defines an $\mathscr{H}(G$, $\Gamma$ )-homomorphism of $\&(\Gamma \backslash X)_{\chi}$ into $\Re$ i.e., the following identity holds for any $f \in \mathscr{H}(G, \Gamma)$ and any $\varphi \in \mathscr{S}(\Gamma \backslash X)_{\chi}$ :

$$
F_{\chi}(f * \varphi)(t)=\hat{f}(t) \cdot F_{\chi}(\varphi)(t) .
$$

Theorem 3. Let $\chi \in \mathfrak{X}^{\text {pr }}$.

(i) The normalized Fourier-Eisenstein transform

$$
F_{\chi}: \&(\Gamma \backslash X)_{\chi} \stackrel{\cong}{\longrightarrow}
$$

is an isomorphism of $\mathscr{H}(G, \Gamma)$-modules.

(ii) The space $\mathscr{S}(\Gamma \backslash X)_{\chi}$ is generated by $c_{\chi, f_{x}}$ as an $\mathscr{H}(G, \Gamma)$-module and we have an $\mathscr{H}(G, \Gamma)$-isomorphism

$$
\mathscr{H}(\Gamma \backslash X)_{\chi} \cong \mathscr{H}(G, \Gamma) / \mathscr{I},
$$

where $\mathscr{I}$ is the ideal of $\mathscr{H}(G, \Gamma)$ generated by $\{T(p, p)-1 \mid p \in \mathbf{P}\}$. 
Proof. It follows from Theorem 2 that $F_{x}$ is bijective. This proves the first part. Since $F_{\chi}\left(c_{\chi, f_{x}}\right)=1$, we get

$$
\mathscr{S}(\Gamma \backslash X)_{\chi}=\mathscr{H}(G, \Gamma) * c_{\chi, f_{\chi}} .
$$

This also implies that $\mathscr{S}(\Gamma \backslash X)_{\chi} \cong \mathscr{H}(G, \Gamma) / \mathscr{I}$, where $\mathscr{I}$ is the kernel of the restricted Satake transform. By the proof of Proposition 4.1 (i), we see that $\mathscr{I}$ is generated by $\{T(p, p)-1 \mid p \in \mathbf{P}\}$.

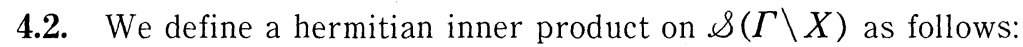

$$
\langle\varphi, \phi\rangle_{\&}=\int_{\tilde{X}} \varphi(x) \overline{\phi(x)} d \mu(x) \quad(\varphi, \phi \in \mathscr{S}(\Gamma \backslash X))
$$

Thus $\mathscr{S}(\Gamma \backslash X)$ becomes a pre-Hilbert space. Let $L^{2}(\Gamma \backslash X)$ be the completion of $\diamond(\Gamma \backslash X)$ :

$$
L^{2}(\Gamma \backslash X)=\left\{\left.\varphi \in \mathscr{C}^{\infty}(\Gamma \backslash X)\left|\sum_{x \in \Gamma \backslash X} \mu(x)\right| \varphi(x)\right|^{2}<+\infty\right\}
$$

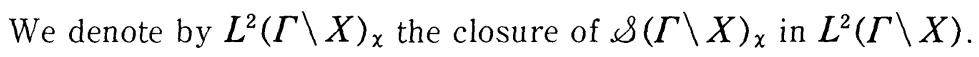

Now we introduce a pre-Hilbert space structure on $\Re$. For $p \in \mathbf{P}$, put

$$
\Re_{p}=\mathbf{C}\left[p^{t}+p^{-t}\right]
$$

Then $\Re$ is canonically isomorphic to the restricted tensor product $\bigotimes_{p \in \mathbf{P}}^{\prime} \Re_{p}$. First we define a hermitian inner product on $\Re_{p}$.

Let $\mathscr{D}_{p}=\sqrt{-1}\left(\mathbf{R} / \frac{2 \pi}{\log p} \mathbf{Z}\right)$ and let $d_{p} t$ be the Haar measure on $\mathscr{D}_{p}$ normalized by $\int_{\mathscr{D}_{p}} d_{p} t=1$. Consider the measure $\omega_{p}(t)$ on $\mathscr{D}_{p}$ given by

$$
\omega_{p}(t)=\frac{1-\chi_{K}(p) p^{-1}}{2} \cdot\left|\frac{L_{p}\left(\chi ; t+\frac{1}{2}\right)}{\zeta_{p}(2 t)}\right|^{2} d_{p} t,
$$

where $\zeta_{p}(2 t)=\frac{1}{1-p^{-2 t}}$. Then we can define an inner product on $\Re_{p}$ by

$$
\left\langle\varphi_{p}, \phi_{p}\right\rangle_{\chi, p}=\int_{\mathscr{D}_{p}} \varphi_{p}(t) \overline{\phi_{p}(t)} \omega_{p}(t) \quad\left(\varphi_{p}, \phi_{p} \in \Re_{p}\right) .
$$

The inner product on $\Re \cong \underset{p \in \mathbf{P}}{\bigotimes^{\prime}} \Re_{p}$ is now defined by

$$
\langle\varphi, \phi\rangle_{\chi}=\frac{\left[\mathscr{O}^{1}: \mathscr{O}_{f_{x}}^{1}\right]}{h_{f_{x}}} \sum_{i, j} a_{i} \bar{b}_{j} \underset{p}{\Pi}\left\langle\varphi_{p, i}, \phi_{p, j}\right\rangle_{x, p}
$$


for

$$
\varphi=\sum_{i} a_{i}\left(\bigotimes_{p} \varphi_{p, i}\right) \text { and } \phi=\sum_{j} b_{j}\left(\bigotimes_{p} \phi_{p, j}\right)\left(a_{i}, b_{j} \in \mathbf{C}, \varphi_{p, i}, \phi_{p, j} \in \Re_{p}\right) .
$$

We denote by $\mathscr{L}_{\chi}^{2}$ (resp. $\mathscr{L}_{\chi, p}^{2}$ ) the completion of $\Re$ (resp. $\Re_{p}$ ) with respect to the inner product $\langle,\rangle_{\chi}\left(\right.$ resp. $\left.\langle,\rangle_{\chi, p}\right)$. The Hilbert space $\mathscr{L}_{\chi}^{2}$ is the Hilbert restricted product of $\mathscr{L}_{\chi, P}^{2}(p \in \mathbf{P})$.

THEOREM 4 (Plancherel formula). The normalized Fourier-Eisenstein transform

$$
F_{\chi}: \&(\Gamma \backslash X)_{\chi} \rightarrow \Re
$$

can be extended to an isometry of $L^{2}(\Gamma \backslash X)_{\chi}$ onto $\mathscr{L}_{\chi}^{2}$. In particular, for every $\varphi, \phi \in$ $\&(\Gamma \backslash X)_{x}$, the following identity holds:

$$
\langle\varphi, \phi\rangle_{\&}=\left\langle F_{\chi}(\varphi), F_{\chi}(\phi)\right\rangle_{\chi} .
$$

First we prove the following result on local factors of the inner product.

Lemma 4.2. For any $p \in \mathbf{P}$, we have

$$
\left\langle\phi_{x, p e}, \phi_{x, p^{d}}\right\rangle_{x, p}=\left\{\begin{array}{cl}
0 & \text { if } d \neq e \\
1 & \text { if } d=e=0 \\
\frac{p^{-e}}{1-\chi_{K, f_{x}}(p) p^{-1}} & \text { if } d=e>0 .
\end{array}\right.
$$

Proof. By (4.6), we have

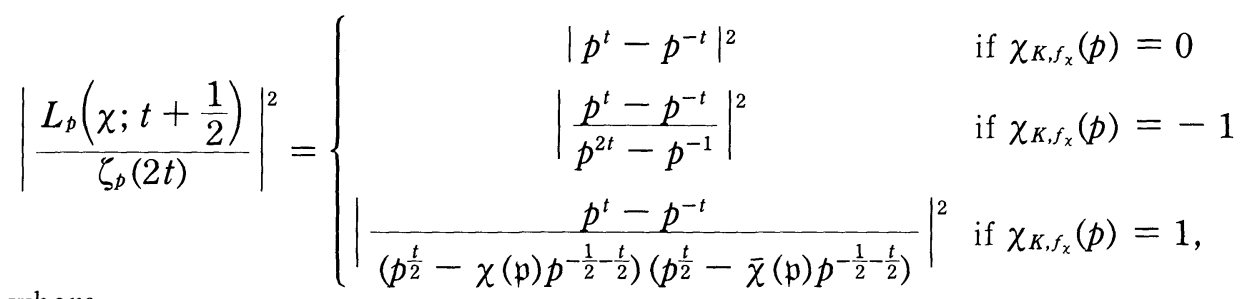

where

$$
\chi(\mathfrak{p})= \begin{cases}\chi([p]) & \text { if } D=1 \\ \chi\left(\left[\mathfrak{p} \cap \mathscr{O}_{f_{x}}\right]\right) & \text { if } D \neq 1 \text { and }(p)=\mathfrak{p} \bar{p} \text { in } K .\end{cases}
$$

Let $\chi_{K, f_{x}}(p)=-1$. Then we get

$$
\int_{\mathscr{D}_{p}} \phi_{x, p^{e}}(t) \overline{\phi_{x, p^{d}}(t)} \omega_{p}(t)
$$




$$
\begin{aligned}
& =\frac{p^{-(d+e) / 2}}{2\left(1+p^{-1}\right)} \int_{\mathfrak{D}_{p}}\left(\frac{p^{(e-1) t}}{p^{-2 t}-p^{-1}}-\frac{p^{-(e-1) t}}{p^{2 t}-p^{-1}}\right) \\
& \times\left\{p^{-(d-1) t}\left(p^{-2 t}-p^{-1}\right)-p^{(d-1) t}\left(p^{2 t}-p^{-1}\right)\right\} d_{p} t \\
& =\frac{p^{-(d+e) / 2}}{2\left(1+p^{-1}\right)} \int_{\mathfrak{D}_{p}}\left(\sum_{l \geq 0} p^{-l+(e+2 l+1) t}-\sum_{m \geq 0} p^{-m-(e+2 m+1) t}\right) \\
& \times\left\{p^{-(d-1) t}\left(p^{-2 t}-p^{-1}\right)-p^{(d-1) t}\left(p^{2 t}-p^{-1}\right)\right\} d_{p} t \\
& =\left\{\begin{array}{cl}
1 & \text { if } d=e=0 \\
\frac{p^{-e}}{1+p^{-1}} & \text { if } d=e>0 \\
0 & \text { if } d \neq e .
\end{array}\right.
\end{aligned}
$$

We can prove the other cases similarly.

Proof of Theorem 4. We have only to show the identity (4.8) for $\varphi=c_{x, e f_{x}}$ and $\phi=c_{\chi, d f_{x}}$ with $e, d \in \mathbf{N}$. It is easy to see that

$$
\left\langle c_{\chi, e f_{x}}, c_{\chi, d f_{x}}\right\rangle_{\mathscr{S}}=\delta_{e, d} \frac{\left[\mathscr{O}^{1}: \mathscr{O}_{e f_{x}}^{1}\right]}{h_{e f_{x}}} .
$$

On the other hand, we get

$$
\left\langle F_{\chi}(\varphi), F_{\chi}(\phi)\right\rangle_{\chi}=\delta_{e, d} \frac{\left[\mathscr{O}^{1}: \mathscr{O}_{f_{\chi}}^{1}\right]}{h_{f_{\chi}}}\left[\mathscr{O}_{f_{\chi}}^{1}: \mathscr{O}_{e f_{\chi}}^{1}\right]^{2} \prod_{p \mid e} \frac{p^{-e_{p}}}{1-\chi_{K, f_{\chi}}(p) p^{-1}},
$$

where $e_{p}=\operatorname{ord}_{p}(e)$. By the class number formula (1.1), we obtain the result.

We define a function $\omega_{x, t}$ in $\mathscr{C}^{\infty}(\Gamma \backslash X) \otimes_{\mathbf{C}} \Re$ by

$$
\omega_{x, t}=\frac{1}{\left[\mathscr{O}^{1}: \mathscr{O}_{f_{x}}^{1}\right]} \sum_{f: f_{x} \mid f} h_{f} \psi_{x, f / f_{x}}(t) c_{\chi, f}
$$

THEOREM 5. For every $\varphi \in \&(\Gamma \backslash X)_{\chi}$, we have

$$
\varphi(x)=\left\langle F_{\chi}(\varphi), \omega_{\bar{\chi}, t}(x)\right\rangle_{\chi},
$$

namely, the inverse transformation of $F_{\chi}$ is given by

$$
F_{\chi}(\varphi) \mapsto\left\langle F_{\chi}(\varphi), \omega_{\bar{\chi}, t}\right\rangle_{\chi}
$$

Proof. By the definition of the inner product $\langle,\rangle_{\&}$, we have 


$$
\varphi(x)=\frac{1}{\mu(x)}\left\langle\varphi, \operatorname{ch}_{x}\right\rangle_{\diamond} .
$$

It is easy to see that

$$
\left\langle p_{x} \varphi, \phi\right\rangle_{\&}=\left\langle\varphi, p_{x} \phi\right\rangle_{\&}
$$

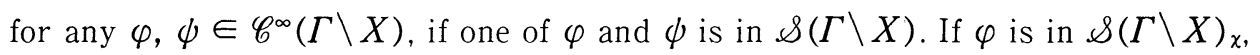
then $\varphi=p_{x} \varphi$. Hence, we get

$$
\varphi(x)=\left\{\begin{array}{cl}
\mu(x)^{-1} \chi\left(p_{f_{\chi}}^{f}([S])\right)\left\langle\varphi, c_{\chi, f}\right\rangle_{\&} & \text { if } f_{\chi} \mid f \\
0 & \text { if } f_{\chi} \nless f,
\end{array}\right.
$$

where $f$ is the conductor of $x$ and $S=f x \in X_{f}^{\mathrm{pr}}$. By Theorem 4, we have

$$
\left\langle\varphi, c_{\chi, f}\right\rangle_{s}=\left\langle F_{\chi}(\varphi), F_{\chi}\left(c_{\chi, f}\right)\right\rangle_{\chi} .
$$

Now the theorem follows immediately from (4.4).

4.3. Theorem 3 enables us to determine all $\mathscr{H}(G, \Gamma)$-common eigenfunctions in $\mathscr{C}^{\infty}(\Gamma \backslash X)$. Since $\Re \cong \bigotimes^{\prime} \Re_{p}$, we can define an algebra homomorphism $\lambda_{\boldsymbol{t}}: \Re \rightarrow \mathbf{C}$ for any $\boldsymbol{t}=\left(t_{p}\right)_{p \in \mathbf{P}} \in \mathbf{C}^{\mathbf{P}}$ by setting

$$
\lambda_{\boldsymbol{t}}\left(\bigotimes_{p} \phi_{p}\right)=\prod_{p \in \mathbf{P}} \phi_{p}\left(t_{p}\right) \quad\left(\phi_{p} \in \Re_{p}, \phi_{p}=1 \text { for almost all } p\right) .
$$

Composing $\lambda_{\boldsymbol{t}}$ with the restricted Satake transform ${ }^{-}: \mathscr{H}(G, \Gamma) \rightarrow \Re$ given by Proposition 4.1 (i), we obtain an algebra homomorphism

$$
\begin{array}{cl}
\mathscr{H}(G, \Gamma) & \rightarrow \mathbf{C} \\
f & \mapsto \hat{f}(\boldsymbol{t}):=\lambda_{\boldsymbol{t}}(\hat{f}) .
\end{array}
$$

Any algebra homomorphism of $\mathscr{H}(G, \Gamma)$ into $\mathbf{C}$ can be obtained in this manner for some $\boldsymbol{t} \in \mathbf{C}^{\mathbf{P}}$.

For $\boldsymbol{t}=\left(t_{p}\right)_{p \in \mathbf{P}} \in \mathbf{C}^{\mathbf{P}}$, define a function $\omega_{x, t} \in \mathscr{C}^{\infty}(\Gamma \backslash X)$ by

$$
\omega_{\chi, t}=\frac{1}{\left[\mathscr{O}^{1}: \mathscr{O}_{f_{x}}^{1}\right]} \sum_{f: f_{x} \mid f} h_{f} \phi_{x, f / f_{x}}(\boldsymbol{t}) c_{\chi, f}
$$

where

$$
\phi_{\chi, f / f_{\chi}}(\boldsymbol{t})=\prod_{p \mid \frac{f}{f_{\chi}}} \phi_{x, p^{e_{p}}}\left(t_{p}\right), \quad e_{p}=\operatorname{ord}_{p}\left(f / f_{\chi}\right) .
$$

It is not hard to check the identity

$$
f * \omega_{x, \boldsymbol{t}}=\hat{f}(\boldsymbol{t}) \omega_{x, \boldsymbol{t}} \quad(f \in \mathscr{H}(G, \Gamma)) .
$$


Theorem 6. Let $\Psi$ be an $\mathscr{H}(G, \Gamma)$-common eigenfunction in $\mathscr{C}^{\infty}(\Gamma \backslash X)$ satisfying

$$
f * \Psi=\hat{f}(\boldsymbol{t}) \Psi \text { for all } f \in \mathscr{H}(G, \Gamma) .
$$

Then $\Psi$ is a (not necessarily finite) linear combination of $\left\{\omega_{\chi, t} \mid \chi \in \mathfrak{X}^{\mathrm{pr}}\right\}$, namely, $\Psi$ is of the form

$$
\Psi=\sum_{\chi} a_{\chi} \cdot \omega_{\chi, t}\left(a_{\chi} \in \mathbf{C}\right) .
$$

Proof. We identify $\mathscr{C}^{\infty}(\Gamma \backslash X)$ with $\operatorname{Hom}_{\mathbf{C}}(\&(\Gamma \backslash X), \mathbf{C})$ via the nondegenerate bilinear form

$$
\begin{aligned}
\langle,\rangle: \mathscr{C}^{\infty}(\Gamma \backslash X) \times \mathscr{S}(\Gamma \backslash X) & \rightarrow \mathbf{C} \\
(\Psi, \varphi) & \mapsto\langle\Psi, \varphi\rangle=\int_{\tilde{X}} \Psi(x) \varphi(x) d \mu(x) .
\end{aligned}
$$

Since $\left\langle p_{\chi}(\Psi), \varphi\right\rangle=\left\langle\Psi, p_{\bar{x}}^{-}(\varphi)\right\rangle$ for any $\Psi \in \mathscr{C}^{\infty}(\Gamma \backslash X)$ and $\varphi \in \mathscr{S}(\Gamma \backslash X)$, the space $\mathscr{C}^{\infty}(\Gamma \backslash X)_{\chi}=p_{\chi}\left(\mathscr{C}^{\infty}(\Gamma \backslash X)\right)$ can naturally be identified with $\operatorname{Hom}_{\mathbf{C}}(\&(\Gamma \backslash$ $\left.X)_{\bar{\chi}}, \mathbf{C}\right)$. By Proposition 2.1 and Theorem 3, we have

$$
\mathscr{C}^{\infty}(\Gamma \backslash X)=\prod_{\chi \in \mathfrak{X}^{\mathrm{pr}}} \mathscr{C}^{\infty}(\Gamma \backslash X)_{\chi} .
$$

Let $\Psi$ be as in the theorem and denote by $\Psi_{\chi}$ the $\mathscr{C}^{\infty}(\Gamma \backslash X)_{\chi}$-component $p_{\chi}(\Psi)$ of $\Psi$. Then, for any $f \in \mathscr{H}(G, \Gamma)$, we have

$$
\begin{aligned}
\left\langle\Psi_{\chi}, f * c_{\bar{\chi}, f_{\chi}}\right\rangle & =\left\langle\Psi, f * c_{\bar{\chi}, f_{\chi}}\right\rangle \\
& =\left\langle f * \Psi, c_{\bar{\chi}, f_{x}}\right\rangle \\
& =\hat{f}(\boldsymbol{t})\left\langle\Psi, c_{\bar{\chi}, f_{\chi}}\right\rangle \\
& =\hat{f}(\boldsymbol{t})\left\langle\Psi_{\chi}, c_{\bar{\chi}, f_{\chi}}\right\rangle .
\end{aligned}
$$

On the other hand, by (4.9), we have

$$
\begin{aligned}
\left\langle\omega_{x, t}, f * c_{\bar{\chi}, f_{x}}\right\rangle & =\hat{f}(\boldsymbol{t})\left\langle\omega_{\chi, \boldsymbol{t}}, c_{\bar{\chi}, f_{x}}\right\rangle . \\
& =\hat{f}(\boldsymbol{t}) \frac{h_{f_{x}}}{\left[\mathscr{O}^{1}: \mathscr{O}_{f_{x}}^{1}\right]} \cdot\left\langle c_{\chi, f_{x}}, c_{\chi, f_{x}}\right\rangle_{\mathscr{S}} \\
& =\hat{f}(\boldsymbol{t}) .
\end{aligned}
$$

Hence

$$
\left\langle\Psi_{\chi}-a_{\chi} \cdot \omega_{\chi, t}, f * c_{\bar{\chi}, f_{\chi}}\right\rangle=0 \quad(f \in \mathscr{H}(G, \Gamma))
$$

where we put

$$
a_{\chi}=\left\langle\Psi_{\chi}, c_{\bar{\chi}, f_{\chi}}\right\rangle
$$


Since $\mathscr{S}(\Gamma \backslash X)_{\chi}=\mathscr{H}(G, \Gamma) * c_{\chi, f_{x}}$ by Theorem 3 , this implies that

$$
\Psi_{\chi}=a_{\chi} \cdot \omega_{\chi, t}
$$

Thus we obtain

$$
\Psi=\sum_{\chi} a_{\chi} \cdot \omega_{\chi, t}
$$

Remark. For the space of nondegenerate binary quadratic forms over $p$-adic fields, results analogous to Theorem 2-6 have been obtained in [H1], [H2].

\section{§5. Examples of Hecke eigenfunctions}

Let $K$ be a real quadratic field with discriminant $D$. As in the previous sections, we put

$$
X=X_{D, 1}=\left\{\left.x \in M(2, \mathbf{Q})\right|^{t} x=x, \operatorname{det} x=-D / 4\right\} .
$$

Put $K^{\prime}=K-\mathbf{Q}$ and consider the bijection

$$
\begin{aligned}
K^{\prime} & \rightarrow X \\
\alpha & \mapsto S_{\alpha}
\end{aligned}
$$

given by

$$
S_{\alpha}=\frac{\sqrt{D}}{\alpha-\overline{\boldsymbol{\alpha}}} \cdot\left(\begin{array}{cc}
1 & -\operatorname{tr}(\alpha) / 2 \\
-\operatorname{tr}(\alpha) / 2 & N(\alpha)
\end{array}\right)
$$

Then, for any $g=\left(\begin{array}{ll}a & b \\ c & d\end{array}\right) \in G=G L_{2}^{+}(\mathbf{Q})$, we have

$$
g * S_{\alpha}=S_{g \cdot \alpha}, \quad g \cdot \alpha=\frac{d \alpha-c}{-b \alpha+a} .
$$

Thus we can identify the space $\mathscr{C}^{\infty}(\Gamma \backslash X)$ with the space

$$
\mathscr{C}^{\infty}\left(\Gamma \backslash K^{\prime}\right)=\left\{\varphi: K^{\prime} \rightarrow \mathbf{C} \mid \varphi(\gamma \cdot \alpha)=\varphi(\alpha)(\gamma \in \Gamma)\right\} .
$$

Hence the Hecke algebra $\mathscr{H}(G, \Gamma)$ acts on $\mathscr{C}^{\infty}\left(\Gamma \backslash K^{\prime}\right)$.

We give examples of Hecke eigenfunctions in $\mathscr{C}^{\infty}\left(\Gamma \backslash K^{\prime}\right)$.

Example 1. In [A], Arakawa introduced the Dirichlet series

$$
\xi(s, \alpha)=\sum_{n=1}^{\infty} \frac{\cot \pi n a}{n^{s}} \quad\left(\alpha \in K^{\prime}\right)
$$


and proved that

(1) $\xi(s, \alpha)$ converges absolutely for $\operatorname{Re} s>1$;

(2) $\xi(s, \alpha)$ has an analytic continuation to a meromorphic function of $s$ on $\mathbf{C}$;

(3) $\xi(s, \alpha)$ has a simple pole at $s=1$.

Let $c_{-1}(\alpha)$ be the residue of $\xi(s, \alpha)$ at $s=1$. Then the following is a reformulation of [A, Theorem 2.16]:

Theorem (Arakawa). The function $c_{-1}(\alpha)$ belongs to $\mathscr{C}^{\infty}\left(\Gamma \backslash K^{\prime}\right)$ and satisfies the identity

$$
f * c_{-1}=\hat{f}\left(-\frac{1}{2}\right) c_{-1} \quad(f \in \mathscr{H}(G, \Gamma))
$$

Example 2. For an $\alpha \in K^{\prime}$, let

$$
\alpha=c_{0}+\frac{1}{c_{1}+\frac{1}{c_{s}+\frac{1}{a_{1}+\frac{1}{a_{k}+\frac{1}{a_{1}+\frac{1}{\ddots}}}}}}
$$

be the expansion into periodic continued fraction. Using the block of periodic terms $a_{1}, \ldots, a_{k}$, we define the Hirzebruch sum $\Psi(\alpha)$ by

$$
\Psi(\alpha)=\left\{\begin{array}{cl}
0 & \text { if } k \text { is odd } \\
\sum_{j=1}^{k}(-1)^{j+s} a_{j} & \text { if } k \text { is even. }
\end{array}\right.
$$

In [Lu], Lu studied the behaviour of $\Psi(\alpha)$ under the action of the Hecke algebra.

Put

$$
\Psi_{0}(\alpha)=\Psi(\alpha) / \mu(\alpha), \quad \mu(\alpha)=\mu\left(S_{\alpha}\right)=\int_{\Gamma * S_{\alpha}} d \mu
$$

Then the following is a reformulation of [ $\mathrm{Lu}$, Theorem 7]:

Theorem $(\mathrm{Lu})$. The function $\Psi_{0}(\alpha)$ belongs to $\mathscr{C}^{\infty}\left(\Gamma \backslash K^{\prime}\right)$ and satisfies the identity

$$
f * \Psi_{0}=\hat{f}\left(-\frac{1}{2}\right) \Psi_{0} \quad(f \in \mathscr{H}(G, \Gamma))
$$


In other words,

$$
f * \Psi=\hat{f}\left(-\frac{1}{2}\right) \Psi \quad(f \in \mathscr{H}(G, \Gamma)) .
$$

Thus the functions $c_{-1}(\alpha)$ and $\Psi_{0}(\alpha)$ belong to the same eigen space of $\mathscr{H}(G$, $\Gamma$ ). Arakawa proved that these two functions essentially coincide with each other.

Proposition (Arakawa).

$$
c_{-1}(\alpha)=-\frac{\pi}{6 \log \varepsilon} \Psi_{0}(\alpha)
$$

where $\varepsilon$ is the totally positive fundamental unit of $K$ with $\varepsilon>\bar{\varepsilon}$.

In $\S 4.3$, we proved that any $\mathscr{H}(G, \Gamma)$-common eigenfunction in $\mathscr{C}^{\infty}(\Gamma \backslash X)$ is a linear combination of $\omega_{x, t}$ 's. If all $t_{p}$ coincide with a fixed $t \in \mathbf{C}$, then, by (4.4), we have

$$
\omega_{\chi, t}=\omega_{\chi, t}=\frac{h_{K}}{2^{1 / 2}\left[\mathscr{O}^{1}: \mathscr{O}_{f_{\chi}}^{1}\right] D^{1 / 4}} \cdot \frac{\left(f_{\chi}\right)^{t+1 / 2}}{L\left(\chi ; t+\frac{1}{2}\right)} \cdot p_{\chi}(E)(x ; t, 0),
$$

where

$$
E(x ; t, 0)=E_{0}(x ; t, 0)+E_{1}(x: t, 0) .
$$

Hence if $L\left(\chi ; t+\frac{1}{2}\right) \neq 0$, eigenfunctions of $\mathscr{H}(G, \Gamma)$ corresponding to the eigenvalue $f \mapsto \hat{f}(t)$ should have an expression in terms of special values of the Eisenstein series (zeta functions of binary quadratic forms) at $(t, 0)$.

For the function $c_{-1}(\alpha)$, such an expression has been obtained by Arakawa, if the conductor of $S_{\alpha}$ is equal to 1 ([A, Proposition 3.1]). Namely, under this assumption, he proved that

$$
c_{-1}(\alpha)=-\frac{2 \pi}{\log \varepsilon} E\left(S_{\alpha} ;-\frac{1}{2}, 0\right) .
$$

By Theorem 6 , the $\mathscr{C}^{\infty}\left(\Gamma \backslash K^{\prime}\right)_{x}$-component of an $\mathscr{H}(G, \Gamma)$-eigenfunction can be determined uniquely up to constant multiple by the corresponding eigenvalue. Hence, by (5.1), we have the following:

THEOREM 7. For any character $\chi$ of $C l_{1}$, the following identity holds:

$$
p_{\chi}\left(c_{-1}\right)(\alpha)=-\frac{2 \pi}{\log \varepsilon} p_{\chi}(E)\left(S_{\alpha} ;-\frac{1}{2}, 0\right) \quad\left(\alpha \in K^{\prime}\right)
$$




\section{REFERENCES}

[A] T. Arakawa, Dirichlet series $\sum_{n=1}^{\infty} \frac{\cot \pi n \alpha}{n^{s}}$, Dedekind sums and Hecke $L$-functions for real quadratic fields, Comment. Math. Univ. St. Pauli, 37 (1988), $209-235$.

[H1] Y. Hironaka, Spherical functions of hermitian and symmetric forms II, Japan. J. Math., 15 (1989), 15-51.

[H2] Y. Hironaka, Spherical functions of hermitian and symmetric forms over 2-adic fields, Comment. Math. Univ. St. Pauli, 39 (1990), 157-193.

[L] S. Lang, Elliptic functions, Addison-Wesley, 1973.

[Lu] H. Lu, Hirzebruch sums and Hecke operators, J. Number Theory, 38 (1991), $185-195$.

[M1] F. I. Mautner, Fonctions propres des opérateurs de Hecke, C. R. Acad. Sci. Paris, Série A 269 (1969), 940-943; 270 (1970), 89-92.

[M2] F. I. Mautner, Spherical functions and Hecke operators, Lie groups and their representations, ed. I. M. Gelfand, Adam Hilger LTD, 1975, pp. 555-576.

[S] F. Sato, Zeta functions in several variables associated with prehomogeneous vector spaces III: Eisenstein series for indefinite quadratic forms, Ann. of Math., 116 (1982), 177-212.

[SH] F. Sato and Y. Hironaka, Eisenstein series on reductive symmetric spaces and representation of Hecke algebras, Preprint, 1991.

[Sn] T. Shintani, On construction of holomorphic cusp forms of half integral weight, Nagoya Math. J., 58 (1975), 83-126.

[Z] D. Zagier, Zeta funktionen und quadratische Körper, Springer-Verlag, 1981.

Yumiko Hironaka

Department of Mathematics

Faculty of Science

Shinshu University

Matsumoto 390

Japan

Fumihiro Sato

Department of Mathematics

Rikkyo University

Nishi-Ikebukuro, Toshimaku, Tokyo 171

Japan 